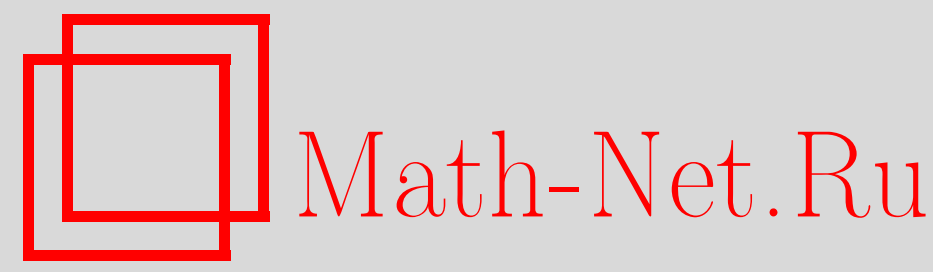

В. В. Жиков, С. Е. Пастухова, Усреднение задач теории упругости на периодических сетках критической толщины, Матем. сб., 2003, том 194, номер 5, 61-96

DOI: https://doi.org/10.4213/sm735

Использование Общероссийского математического портала Math-Net.Ru подразумевает, что вы прочитали и согласны с пользовательским соглашением

http://www . mathnet.ru/rus/agreement

Параметры загрузки:

IP : 107.22 .136 .117

26 апреля 2023 г., 15:06:55 


\section{Усреднение задач теории упругости на периодических сетках критической толщины}

Существенной особенностью задач теории упругости на периодических структурах, зависящих от двух геометрических параметров, является неклассический характер их усреднения. Самый сложньй вид неклассического усреднения возникает на структурах так называемой критической толщины. В статье излагается усреднение периодических сеток этого типа.

Библиографоия: 13 названий.

\section{§1. Введение}

1.1. На рис. 1 изображена периодическая сетка или стержневая структура, составленная из стержней толщины $2 h>0$, ячейка периодичности $\square=\left[-\frac{1}{2}, \frac{1}{2}\right)^{2}$ указана пунктиром. Там же изображена и бесконечно тонкая сетка, отвечающая толщине $h=0$. Данная сетка есть лишь модельный пример. Наши результаты справедливы и для более сложно устроенных сеток, не обладающих столь богатой симметрией, как эта.
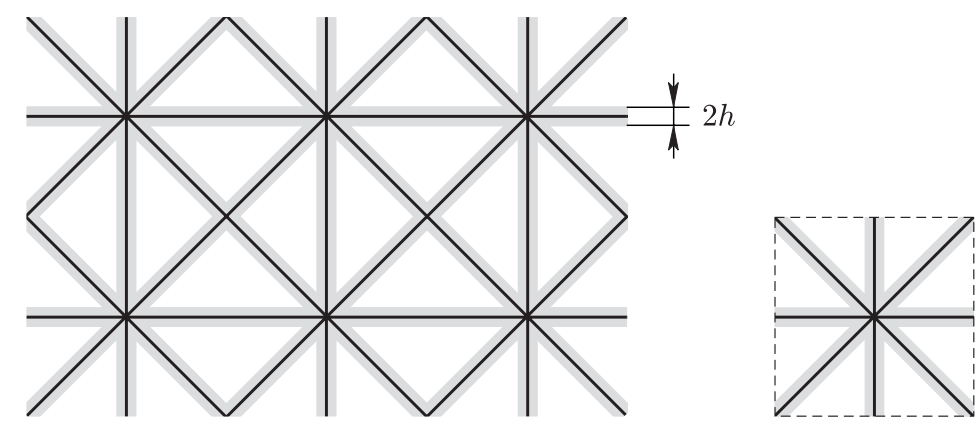

Рис. 1. Периодическая сетка или стержневая структура

Введем некоторые обозначения.

Пусть $F^{h}$ - периодическая сетка толщины $2 h>0 ; F_{\varepsilon}^{h}=\varepsilon F^{h}$-гомотетическое сжатие сетки $F^{h} ; F$ - бесконечно тонкая или сингулярная сетка; $\mu$-периодическая

Работа выполнена при финансовой поддержке Российского фонда фундаментальныт исследований (грант № 02-01-00114). 
нормированная мера, сосредоточенная на сингулярной сетке $F$ и пропорциональная там одномерной мере Лебега,

$$
\langle g\rangle=\int_{\square} g d \mu
$$

- среднее по мере $\mu$.

Через $A=\left\{a_{i j s p}\right\}$ обозначим тензор упругости, подчиненный обычным условиям симметрии: $a_{i j s p}=a_{s p i j}=a_{j i s p}$. Скалярное произведение симметрических матриц $\xi=\left\{\xi_{i j}\right\}, \eta=\left\{\eta_{i j}\right\}$ определим как $\xi \cdot \eta=\xi_{i j} \cdot \eta_{i j}$, в частности, $\xi \cdot \xi=\xi^{2}$. Действие тензора $A$ на матрищу $\xi$ есть матрица $A \xi=\left\{a_{i j s p} \xi_{s p}\right\}$. Тогда $A \xi \cdot \xi=a_{i j s p} \xi_{i j} \xi_{s p}-$ плотность упругой энергии. Предполагаем, что тензор $A$ положительно определен, $A \xi \cdot \xi \geqslant c_{0} \xi^{2}, c_{0}>0$.

Для изотропного тензора имеем

$$
\begin{gathered}
A \xi=k \xi+k_{1} E \operatorname{tr} \xi, \quad k>0, \quad k_{1} \geqslant 0, \\
\xi=\left(\begin{array}{ll}
\xi_{11} & \xi_{12} \\
\xi_{12} & \xi_{22}
\end{array}\right), \quad \operatorname{tr} \xi=\xi_{11}+\xi_{22}, \quad E=\left(\begin{array}{ll}
1 & 0 \\
0 & 1
\end{array}\right) .
\end{gathered}
$$

С ограниченной липшицевой областью $\Omega \subset \mathbb{R}^{2}$ свяжем перфорированную область $\Omega \cap F_{\varepsilon}^{h}$ и пространство $W_{\varepsilon, h}$ - замькание множества $C_{0}^{\infty}(\Omega)^{2}$ по норме

$$
\left(\int_{\Omega \cap F_{\varepsilon}^{h}}(\varphi \cdot \varphi+e(\varphi) \cdot e(\varphi)) d x\right)^{1 / 2}
$$

$e(\varphi)=\frac{1}{2}\left\{\frac{\partial \varphi_{i}}{\partial x_{j}}+\frac{\partial \varphi_{j}}{\partial x_{i}}\right\}-$ тензор деформации.

Рассмотрим задачу: найти вектор-функиию $u^{\varepsilon, h} \in W_{\varepsilon, h}$, для которой въ-полнено интегральное тождество

$$
\begin{gathered}
\int_{\Omega \cap F_{\varepsilon}^{h}}\left(A e\left(u^{\varepsilon, h}\right) \cdot e(\varphi)+u^{\varepsilon, h} \cdot \varphi\right) d x=\int_{\Omega \cap F_{\varepsilon}^{h}} f \cdot \varphi d x \\
\forall \varphi \in C_{0}^{\infty}(\Omega)^{2}, \quad f \in C^{\infty}(\bar{\Omega})^{2} .
\end{gathered}
$$

Это - обобщенная или вариационная формулировка краевой задачи для системы теории упругости в области $\Omega \cap F_{\varepsilon}^{h}$, когда на $\partial \Omega \cap \partial F_{\varepsilon}^{h}$ задается условие закрепления, т.е. условие Дирихле $u^{\varepsilon, h}=0$, а на остальной части границы области $\Omega \cap F_{\varepsilon}^{h}$ - условие отсутствия напряжений $A e\left(u^{\varepsilon, h}\right) n=0, n$ - нормаль к гранище.

Хорошо известно [1], что решение поставленной задачи сушествует и единственHо.

Цель усреднения состоит в том, чтобы изучить поведение решения $u^{\varepsilon, h}$ при $\varepsilon \rightarrow 0$ и найти уравнение, которому удовлетворяет предельная функция. Случай, когда толщина $h>0$ фиксирована, охватывается классической теорией усреднения в перфорированных областях (см. [1]-[3]). Будем считать, что толщина стержней стремится к нулю вместе с $\varepsilon$, т.е. $h=h(\varepsilon) \rightarrow 0$ при $\varepsilon \rightarrow 0$. И такая постановка вопроса не является новой. Например, для скалярных задач в аналогичной ситуации доказана “сильная" сходимость

$$
\lim _{\varepsilon \rightarrow 0} \frac{1}{\left|\Omega \cap F_{\varepsilon}^{h}\right|} \int_{\Omega}\left|u^{\varepsilon, h}(x)-u^{0}(x)\right|^{2} d x=0,
$$


где $u^{0}-$ решение усредненной задачи Дирихле

$$
u^{0} \in H_{0}^{1}(\Omega), \quad-\operatorname{div} A^{\mathrm{hom}} \nabla u^{0}+u^{0}=f .
$$

При этом усредненная матрица $A^{\text {hom }}$ определяется с помошью некоторой периодической задачи на сингулярной сетке $F$. Важно, что предел решений $u^{\varepsilon, h}$ не зависит от способа стремления $h$ к нулю [1; гл. 8].

Для задач теории упругости обнаружен (см. [4], [5]) "масштабный эффект": усредненное уравнение существенно зависит от того, как толщина $h$ стремится к нулю при $\varepsilon \rightarrow 0$.

Были выделены три случая:

i) достаточно толстые сетки, когда $\lim _{\varepsilon \rightarrow 0} h(\varepsilon) / \varepsilon=\infty$;

ii) достаточно тонкие сетки, когда $\lim _{\varepsilon \rightarrow 0} h(\varepsilon) / \varepsilon=0$;

iii) сетки критической толщины, когда

$$
\lim _{\varepsilon \rightarrow 0} \frac{h(\varepsilon)}{\varepsilon}=\theta>0 .
$$

Особенностью задач теории упругости является то, что решение $u^{\varepsilon, h}$ “осциллирует" и для него, вообше говоря, не может быть сильной сходимости (1.3). Более точно, в нулевом приближении решение $u^{\varepsilon, h}(x)$ не есть гладкая функция $u^{0}(x)$, а имеет вид $u(x, x / \varepsilon)$, где $u(x, y)$ - функция двух переменных, периодическая по аргументу $y$. Функция $u(x, y)$ служит “двухмасштабным пределом" последовательности $u^{\varepsilon, h}$. При этом структура функции $u(x, y)$ и уравнение, которому она удовлетворяет, сушественно различаются в указанных выше трех случаях, хотя всегда $u(x, y)$ как функция аргумента $y$ есть “периодическое жесткое перемешение на сингулярной сетке $F$ ".

Поясним это ключевое понятие. Скажем, что заданный на сингулярной сетке $F$ вектор $u \in L^{2}(\square, d \mu)^{2}$ есть периодическое жсесткое перемещение, если найдется последовательность гладких периодических векторов $\varphi_{\delta} \in C_{\mathrm{per}}^{\infty}(\square)^{2}$ такая, что

$$
\varphi_{\delta} \rightarrow u, \quad e\left(\varphi_{\delta}\right) \rightarrow 0 \quad \text { в } \quad L^{2}(\square, d \mu)
$$

Множество периодических жестких перемещений будем обозначать через $\mathscr{R}$. Доказано, что всякий вектор $u \in \mathscr{R}$ допускает единственное представление

$$
u(y)=c+\chi(y)
$$

в котором $c$ - постоянный вектор, а $\chi$ - поперечное перемешение. Последнее означает, что на каждом звене сетки $F$ вектор $\chi$ ортогонален этому звену. Таким образом, справедливо разложение $\mathscr{R}=\mathbb{R}^{2}+\mathscr{R}_{1}$, где $\mathscr{R}_{1}-$ множество всех поперечных перемещений.

Ортогональный проектор $P_{1}: \mathscr{R} \rightarrow \mathscr{R}_{1}$ переводит постоянный вектор $c \in \mathbb{R}^{2}$ в поперечное перемешение, которое на каждом звене равно нормальной компоненте этого вектора. Результат записьвается как $P_{1}(y) c$. В выражении вида $P_{1}(y) f(x)$ вектор $f$ рассматривается как постоянный, $x$ играет роль параметра.

Чтобы лучше понять проблемы критического случая, сформулируем некоторые результаты, справедливые при любом $h(\varepsilon) \rightarrow 0$. 
1) Найдется вектор-функция $u(x, y) \in L^{2}(\Omega \times \square, d x \times d \mu)^{2}$, периодическая по $y$, такая, что

$$
\lim _{\varepsilon \rightarrow 0} \frac{1}{\left|\Omega \cap F_{\varepsilon}^{h}\right|} \int_{\Omega \cap F_{\varepsilon}^{h}}\left|u^{\varepsilon, h}(x)-u\left(x, \varepsilon^{-1} x\right)\right|^{2} d x=0 .
$$

Здесь требуется пояснение, так как функция $u(x, \cdot)$ задана на сингулярной сетке $F$, а для интегрирования в (1.6) нужно, чтобы она была определена на сетке $F^{h}$. Достаточно продолжить эту функцию как постоянную в поперечном направлении на каждый стержень, а в точках, принадлежащих двум или более стержням, определить ее как сумму таких продолжений (“естественное" продолжение, см. $\S 2$ ).

$2)$ Вектор $u(x, \cdot)$ есть периодическое жесткое перемешение и в соответствии с разложением (1.5)

$$
u(x, y)=u^{0}(x)+\chi(x, y), \quad \chi(x, \cdot) \in \mathscr{R}_{1} \quad \text { для п.в. } x \in \Omega .
$$

3) Вьполнено соотношение

$$
u^{0} \in H_{0}^{1}(\Omega)^{2}, \quad-\operatorname{div} A^{\mathrm{hom}} e\left(u^{0}\right)+u^{0}+\langle\chi\rangle=f,
$$

в котором $A^{\text {hom }}$ - усредненный тензор,

$$
A^{\text {hom }} \xi \cdot \xi=\inf _{w \in C_{\text {per }}^{\infty}(\square)^{2}} \int_{\square} A(\xi+e(w)) \cdot(\xi+e(w)) d \mu .
$$

Для модельной сетки тензор $A^{\text {hom }}$ положительно определенный: $A^{\text {hom }} \xi \cdot \xi \geqslant c_{0} \xi^{2}$, $c_{0}>0$.

Соотношения (1.7), (1.8) нельзя рассматривать как усредненное уравнение, поскольку они не определяют однозначно саму функцию $u(x, y)$. Необходимо найти дополнительную связь между компонентами $u^{0}$ и $\chi$. Для достаточно толстых сеток эта связь имеет тривиальный вид $\chi=0$, и получаем классическое усреднение

$$
\begin{gathered}
\lim _{\varepsilon \rightarrow 0} \frac{1}{\left|\Omega \cap F_{\varepsilon}^{h}\right|} \int_{\Omega \cap F_{\varepsilon}^{h}}\left|u^{\varepsilon, h}(x)-u^{0}(x)\right|^{2} d x=0, \\
u^{0} \in H_{0}^{1}(\Omega)^{2}, \quad-\operatorname{div} A^{\text {hom }} e\left(u^{0}\right)+u^{0}=f .
\end{gathered}
$$

Для достаточно тонких сеток дополнительная связь также известна:

$$
u^{0}(x)+\chi(x, \cdot)-f(x) \perp \mathscr{R}_{1} .
$$

Отсюда и из (1.8) получаем

$$
\begin{gathered}
u^{0} \in H_{0}^{1}(\Omega)^{2}, \quad-\operatorname{div} A^{\mathrm{hom}} e\left(u^{0}\right)+T u^{0}=T f, \quad T=E-\left\langle P_{1}\right\rangle, \\
\chi(x, y)=P_{1}(y)\left(f(x)-u^{0}(x)\right) .
\end{gathered}
$$

Для модельной сетки $T=\frac{1}{2} E$.

В настоящей работе мы находим дополнительную связь между компонентами $u^{0}$ и $\chi$ в случае сеток критической толшины. Оказывается, что компонента $\chi(x, y)$ как функция аргумента $y$ удовлетворяет на каждом звене уравнению четвертого порядка; эти уравнения содержат "пространственную" компоненту $u^{0}$ в качестве параметра и связаны между собой некоторыми условиями сопряжения в узлах. Взятые вместе, они дают хорошо поставленную периодическую задачу на сингулярной сетке. Ниже эта задача будет описана. 
1.2. Рассмотрим отдельное звено сетки $F$ - отрезок $I$ на плоскости. Пусть $\tau-$ направление вдоль звена (одно из двух возможных), $\nu$ - нормаль к звену, $\tau, \nu$ правая пара ортов, $\chi$ - поперечное перемещение на сетке.

Очевидно, что производная $d(\chi \cdot \nu) / d \tau$ не зависит от выбора $\tau$; обозначим ее через $(\chi \cdot \nu)^{\prime}$. Аналогично вектор $\nu d^{2}(\chi \cdot \nu) / d \tau^{2}$ не зависит от выбора направления и обозначается $\chi^{\prime \prime}$. Видим, что инвариантно определены нечетные производные проекции: $(\chi \cdot \nu)^{\prime},(\chi \cdot \nu)^{\prime \prime \prime}, \ldots$, а также четные производные вектора: $\chi^{\prime \prime}, \chi^{\mathrm{IV}}, \ldots$

Пусть в узле $O$ сходятся звенья $I_{1}, I_{2}, \ldots, I_{m}$. Нас будут интересовать следующие свойства.

а) Функция $\left.\chi \cdot \nu_{j}\right|_{I_{j}}, j=1, \ldots, m$, имеет первую и вторую производные, суммируемые с квадратом на отрезке $I_{j}$, или коротко $\chi \cdot \nu_{j} \in H^{2}\left(I_{j}\right)$.

В этом случае первая производная $(\chi \cdot \nu)^{\prime}$ непрерывна на замкнутом отрезке $I_{j}$ и можно говорить о ее значении в узле.

b) Равенство первых производных в узле:

$$
\left.\left(\chi \cdot \nu_{1}\right)^{\prime}\right|_{O}=\left.\left(\chi \cdot \nu_{2}\right)^{\prime}\right|_{O}=\cdots=\left.\left(\chi \cdot \nu_{m}\right)^{\prime}\right|_{O}
$$

с) Закрепление в узле: $\left.\chi\right|_{O}=0$.

Некоторого пояснения требует, быть может, свойство (1.11).

Рассмотрим пример двух отрезков $I_{1}, I_{2}$, выходящих из начала координат в направлении координатных осей. Поскольку вектор $\chi=\left(\chi_{1}, \chi_{2}\right)$ есть поперечное перемешение на $I_{1} \cup I_{2}$, то компонента $\chi_{1}$ отлична от нуля только на $I_{2}$ (где ее запишем как $\left.\chi_{1}\left(y_{2}\right)\right)$, а $\chi_{2}$ отлична от нуля только на $I_{1}$ (где запишем ее как $\left.\chi_{2}\left(y_{1}\right)\right)$. Тогда свойство (1.11) означает равенство

$$
\frac{d \chi_{1}(0)}{d y_{2}}+\frac{d \chi_{2}(0)}{d y_{1}}=0 .
$$

Возьмем совсем простой пример, когда отрезки $I_{1}, I_{2}$ лежат на одной прямой, скажем, на оси $O y_{1}$, а “узлом" служит начало координат. Тогда $\chi=\left\{0, \chi_{2}\left(y_{1}\right)\right\}$ и условие (1.11) означает равенство левой и правой производных функции $\chi_{2}$ в точке $y_{1}=0$.

ОПРЕДЕЛЕНИЕ 1.1. Пусть $\mathscr{R}_{1}^{0} \subset \mathscr{R}_{1}$ - это множество всех периодических поперечных перемешений, удовлетворяющих условиям а)-c) на каждом звене и в каждом узле. Норму в $\mathscr{R}_{1}^{0}$ определим как сумму $H^{2}$-норм по всем звеньям.

Теперь можно описать периодическую задачу на сингулярной сетке. Если исходньй тензор $A$ изотропен (см. (1.1)), то вектор-функция $\chi=\chi(x, \cdot)$ есть решение вариационной задачи на отыскание минимума

$$
\begin{gathered}
\min _{\chi \in \mathscr{R}_{1}^{0}} \int_{\square}\left(\frac{\theta^{2}}{3} \widehat{k}\left|\chi^{\prime \prime}\right|^{2}+|\chi|^{2}-2 \chi \cdot P_{1} c\right) d \mu, \\
\widehat{k}=\frac{k\left(k+2 k_{1}\right)}{k+k_{1}}, \quad c=f(x)-u^{0}(x) .
\end{gathered}
$$

Здесь $\theta$ - параметр толщины сетки (см. (1.4)), $k, k_{1}$ - модули упругости из (1.1). 
Для решения этой вариационной задачи выполнена слабая форма уравнения Эйлера

$$
\chi \in \mathscr{R}_{1}^{0}, \quad \int_{\square}\left(\frac{\theta^{2}}{3} \widehat{k} \chi^{\prime \prime} \cdot v^{\prime \prime}+\chi \cdot v-v \cdot P_{1} c\right) d \mu=0 \quad \forall v \in \mathscr{R}_{1}^{0},
$$

из которой вытекает собственно уравнение Эйлера

$$
\frac{\theta^{2}}{3} \widehat{k} \chi^{\mathrm{IV}}+\chi=P_{1} c, \quad c=f(x)-u^{0}(x),
$$

и для каждого узла - "естественное" условие

$$
\left.\sum \frac{d^{2}\left(\chi \cdot \nu_{j}\right)}{d \tau_{j}^{2}}\right|_{O}=0
$$

где все направления $\tau_{j}$ выбраны выходящими из узла (или входящими в узел).

Часто условия в узлах можно упростить, воспользовавшись симметрией. Например, модельная сетка инвариантна относительно поворота на $180^{\circ}$ вокруг любого из своих узлов. Поэтому решение вариационной задачи (1.12) также обладает указанной симметрией и условие (1.11) выглядит так: производные $\left.\left(\chi \cdot \nu_{j}\right)^{\prime}\right|_{O}$ равны нулю. Другими словами, все звенья сингулярной сетки жестко закреплены в узлах, а естественное условие (1.14) выполнено автоматически. Периодическая задача сводится к стандартным краевым задачам на каждом звене в отдельности.

Уравнение (1.13) на сетке $F$, дополненное условиями в узлах, однозначно разрешимо. Решение можно записать в виде $\chi=\omega(y) P_{1} c$, где $\omega(y)=\omega(y, \theta)$ - некоторая периодическая функция на сингулярной сетке. Поскольку $c=f(x)-u^{0}(x)$, то из (1.8) получаем

$$
\begin{gathered}
-\operatorname{div} A^{\mathrm{hom}} e\left(u^{0}\right)+S u^{0}=S f, \quad S=E-\left\langle\omega P_{1}\right\rangle, \\
\chi(x, y)=\omega(y) P_{1}(y)\left(f(x)-u^{0}(x)\right),
\end{gathered}
$$

причем матрица $S$ удовлетворяет неравенству $T<S<E$, а для модельной сетки она пропорциональна единичной. Первое из полученных соотношений дает отдельную эллиптическую задачу для компоненты $u^{0}(x)$, а второе - связь между компонентами $u^{0}$ и $\chi$. Функция $\omega$ зависит от параметра $\theta$, при этом

$$
\lim _{\theta \rightarrow \infty} S=E, \quad \lim _{\theta \rightarrow \infty} \omega P_{1}=0, \quad \lim _{\theta \rightarrow 0} S=T, \quad \lim _{\theta \rightarrow 0} \omega P_{1}=P_{1}
$$

Видим, что при $\theta \rightarrow \infty$ уравнение (1.15) переходит в классическое усредненное уравнение (1.9), а при $\theta \rightarrow 0$ переходит в неклассическое уравнение (1.10).

1.3. Дадим более формальное описание усредненной задачи. 
ОПРЕДЕЛЕНИЕ 1.2. Пусть $V$ - множество вектор-функций вида

$$
u=u^{0}(x)+\chi(x, y), \quad u^{0} \in H_{0}^{1}(\Omega)^{2}, \quad \chi \in L^{2}\left(\Omega, \mathscr{R}_{1}^{0}\right) .
$$

Скажем, что $u \in V$ есть решение усредненной задачи, если интегральное тождество

$$
\begin{aligned}
\int_{\Omega} A^{\mathrm{hom}} e\left(u^{0}\right) \cdot e\left(\varphi^{0}\right) d x & +\frac{\theta^{2}}{3} \int_{\Omega} \int_{\square} \widehat{k} \chi^{\prime \prime} \cdot \psi^{\prime \prime} d x d \mu+\int_{\Omega} \int_{\square} u \cdot \varphi d x d \mu \\
& =\int_{\Omega} \int_{\square} f \cdot \varphi d x d \mu
\end{aligned}
$$

вьполнено для любой вектор-функции $\varphi=\varphi^{0}+\psi \in V$.

Множество $V$ назовем әнергетическим пространством усредненной задачи.

Полагая в этом тождестве $\psi=0$, получаем соотношение (1.8). Далее, взяв $\varphi^{0}=0, \psi=\eta(x) v(y)$, где $\eta \in C_{0}^{\infty}(\Omega), v \in \mathscr{R}_{1}^{0}$, получим тождество

$$
\int_{\square}\left(\frac{\theta^{2}}{3} \widehat{k} \chi^{\prime \prime} \cdot v^{\prime \prime}+\left(u^{0}+\chi-f\right) \cdot v\right) d \mu(y)=0 \quad \forall v \in \mathscr{R}_{1}^{0} .
$$

Вектор $u^{0}+\chi-f$ можно заменить вектором $\chi+P_{1}\left(u^{0}-f\right)$ и вывести из этого тождества уравнение (1.13) и естественное условие (1.14).

Усредненное уравнение в форме тождества (1.17) более удобно в некоторых вопросах. Например, полагая в этом тождестве $\psi=u$, получаем энергетическое равенство

$$
\begin{aligned}
\int_{\Omega} A^{\text {hom }} e\left(u^{0}\right) \cdot e\left(u^{0}\right) d x & +\frac{\theta^{2}}{3} \int_{\Omega} \int_{\square} \widehat{k} \chi^{\prime \prime} \cdot \chi^{\prime \prime} d x d \mu+\int_{\Omega} \int_{\square} u \cdot u d x d \mu \\
& =\int_{\Omega} \int_{\square} f \cdot u d x d \mu
\end{aligned}
$$

Отметим, что в обоих некритических случаях второе слагаемое слева отсутствует. В работе [5] было доказано, что в критическом случае энергетический баланс требует, чтобы на втором месте слева стояло некоторое положительное слагаемое, но точный вид этого слагаемого не был найден.

Сформулируем основной результат по усреднению в критическом случае.

ТЕОрема 1.3. Пусть $u^{\varepsilon, h}$ - решение задачи (1.2), причем тензор А изотропен. Тогда имеет место соотношение $(1.6)$, в котором $u(x, y)=u^{0}(x)+$ $\chi(x, y)$ есть решение усредненной задачи (1.17). Имеет место такжсе сходимость упругих энергий

$$
\begin{aligned}
& \lim _{\varepsilon \rightarrow 0} \frac{1}{\left|\Omega \cap F_{\varepsilon}^{h}\right|} \int_{\Omega \cap F_{\varepsilon}^{h}} A e\left(u^{\varepsilon, h}\right) \cdot e\left(u^{\varepsilon, h}\right) d x \\
& \quad=\int_{\Omega} A^{\text {hom }} e\left(u^{0}\right) \cdot e\left(u^{0}\right) d x+\frac{\theta^{2}}{3} \int_{\Omega} \int_{\square} \widehat{k} \chi^{\prime \prime} \cdot \chi^{\prime \prime} d x d \mu .
\end{aligned}
$$


1.4. Выше предполагалось, что исходный тензор $A$ изотропен. В случае тензора $A$ общего вида усредненное уравнение немного усложняется, в частности, аналог вариационной задачи (1.12) имеет вид

$$
\min _{\chi \in \mathscr{R}_{1}^{0}} \int_{\square}\left(\frac{\theta^{2}}{3} \rho\left|\chi^{\prime \prime}\right|^{2}+|\chi|^{2}-2 \chi \cdot P_{1} c\right) d \mu, \quad c=f(x)-u^{0}(x),
$$

где $\rho=\rho(y)$ - периодическая функция на сетке $F$, постоянная на каждом звене,

$$
\rho=\left(A^{-1} \eta \cdot \eta\right)^{-1}, \quad \eta=\tau \times \tau, \quad \tau-\text { направление звена. }
$$

Напомним, что по определению

$$
\tau \times \tau=\left\{\tau^{i} \tau^{j}\right\}
$$

Для изотропного тензора $A$ (см. (1.1)) функция $\rho$ вычисляется в явном виде. Действительно, в этом случае нетрудно найти обратньй тензор:

$$
\begin{aligned}
& A^{-1} \eta=\frac{\eta}{k}-\frac{k_{1}}{k\left(k+2 k_{1}\right)} E \operatorname{tr} \eta \\
& A^{-1} \eta \cdot \eta=\frac{\eta \cdot \eta}{k}-\frac{k_{1}(\operatorname{tr} \eta)^{2}}{k\left(k+2 k_{1}\right)}
\end{aligned}
$$

где $\eta$ - произвольная симметрическая матрица. Для матрицы $\eta=\tau \times \tau$ имеем $\eta \cdot \eta=1, \operatorname{tr} \eta=1$ и поэтому $A^{-1} \eta \cdot \eta=1 / \widehat{k}$ не зависит от направления стержня. Видим, что в изотропном случае $\rho=\widehat{k}$, и приходим к вариационной задаче (1.12).

Для тензора $A$ общего вида теорема усреднения сохраняется, нужно только в $(1.17)$ и (1.19) заменить $\widehat{k}$ на $\rho$.

Еще одно замечание. Модельная сингулярная сетка составлена из четырех периодически повторяющихся бесконечных прямых. Аналогичную структуру имеет и тонкая сетка $F^{h}$. Представляют интерес и более сложно устроенные сингулярные сетки, которые нельзя составить из бесконечных прямых (см. рис. 2). В таком случае следует оговорить, как строится соответствующая тонкая сетка $F^{h}$. Звено сетки $F$ - это принадлежащий $F$ отрезок, концы которого служат узлами. Для каждого звена $I$ строим полосу $I^{h}$ ширины $2 h$ со средней линией $I$. Тогда сетка $F^{h}$ определяется как объединение всех построенных полос. Иногда удобно добавлять сюда еше и круги радиуса $h$ с центрами в узлах.

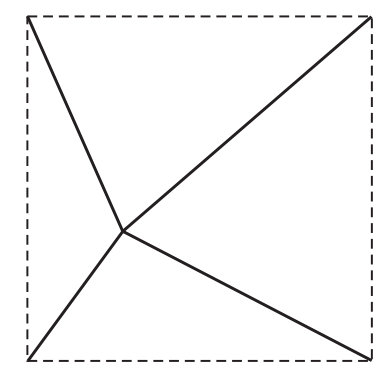

Рис. 2. 
Теорема 1.3 сохраняется для сеток $F^{h}$ весьма обшего вида. Минимальные требования таковы: сингулярная сетка $F$ должна быть связной на торе периодичности и содержать хотя бы один узел, т.е. по крайней мере два неколлинеарных звена.

1.5. Затронем важньй вопрос о справедливости теоремы усреднения для задачи теории упругости в статической постановке, т.е. для задачи

$$
u^{\varepsilon, h} \in W_{\varepsilon, h}, \quad \int_{\Omega \cap F_{\varepsilon}^{h}} A e\left(u^{\varepsilon, h}\right) \cdot e(\varphi) d x=\int_{\Omega \cap F_{\varepsilon}^{h}} f \cdot \varphi d x \quad \forall \varphi \in C_{0}^{\infty}(\Omega)^{2} .
$$

Для того чтобы эта задача могла быть исследована теми же методами, что и исходная задача (1.2), требуется неравенство Корна

$$
\int_{\Omega \cap F_{\varepsilon}^{h}} \varphi \cdot \varphi d x \leqslant c \int_{\Omega \cap F_{\varepsilon}^{h}} e(\varphi) \cdot e(\varphi) d x, \quad \varphi \in C_{0}^{\infty}(\Omega)^{2}
$$

в котором постоянная $c$ не зависит от $\varphi, \varepsilon, h$.

Неравенство Корна для модельной сетки доказано в $\S 5$. В этом случае усредненное уравнение получается из (1.17) вычеркиванием третьего слагаемого слева, т.е. принимает вид

$$
\begin{gathered}
u \in V, \int_{\Omega} A^{\text {hom }} e\left(u^{0}\right) \cdot e\left(\varphi^{0}\right) d x+\frac{\theta^{2}}{3} \int_{\Omega} \int_{\square} \widehat{k} \chi^{\prime \prime} \cdot \psi^{\prime \prime} d x d \mu=\int_{\Omega} \int_{\square} f \cdot \varphi d x d \mu \\
\forall \varphi=\varphi^{0}+\psi \in V .
\end{gathered}
$$

Это уравнение для вектор-функции $u=u^{0}+\chi$ распадается на отдельные уравнения для компонент $u^{0}$ и $\chi$. Компонента $u^{0}$ есть решение “обычной” усредненной задачи

$$
u^{0} \in H_{0}^{1}(\Omega)^{2}, \quad-\operatorname{div} A^{\text {hom }} e\left(u^{0}\right)=f \quad \text { в } \Omega .
$$

Компонента $\chi=\chi(x, \cdot)$ удовлетворяет уравнению

$$
\chi \in \mathscr{R}_{1}^{0}, \quad \frac{\theta^{2}}{3} \widehat{k} \chi^{\mathrm{IV}}=P_{1} f
$$

на сингулярной сетке $F$, дополненному естественными краевьми условиями (1.14) в каждом узле.

Формулировка самой теоремы усреднения полностью сохраняется (подробнее cM. $\S 4)$.

\section{§ 2. Предварительные сведения}

2.1. В настоящей работе применяется так называемый "measure approach", coединенный с техникой двухмасштабной сходимости. Сейчас мы отметим только начальные моменты этого подхода.

Пусть $\mu^{h}$ - периодическая нормированная мера на $\mathbb{R}^{2}$, сосредоточенная на сетке $F^{h}$ и пропорциональная там плоской мере Лебега. Легко видеть, что

$$
d \mu^{h} \rightarrow d \mu, \quad h \rightarrow 0
$$


где $\mu$-мера на сингулярной сетке. Подробнее это означает, что

$$
\lim _{h \rightarrow 0} \int_{\square} \varphi d \mu^{h}=\int_{\square} \varphi d \mu \quad \forall \varphi \in C_{\text {per }}^{\infty}(\square) .
$$

Определим $\varepsilon$-периодическую меру $\mu_{\varepsilon}^{h}$ равенством

$$
\mu_{\varepsilon}^{h}(B)=\varepsilon^{2} \mu^{h}\left(\varepsilon^{-1} B\right)
$$

для любого борелева множества $B \subset \mathbb{R}^{2}$. Очевидно, что мера $\mu_{\varepsilon}^{h}$ сосредоточена на сжатой сетке $F_{\varepsilon}^{h}$. Свяжем параметры $h$ и $\varepsilon$, полагая, что $h=h(\varepsilon) \rightarrow 0$.

В терминах меры $\mu_{\varepsilon}^{h}$ задача (1.2) записывается следующим образом:

$u^{\varepsilon, h} \in W_{\varepsilon, h}, \quad \int_{\Omega}\left(A e\left(u^{\varepsilon, h}\right) \cdot e(\varphi)+u^{\varepsilon, h} \cdot \varphi\right) d \mu_{\varepsilon}^{h}=\int_{\Omega} f^{\varepsilon, h} \cdot \varphi d \mu_{\varepsilon}^{h} \forall \varphi \in C_{0}^{\infty}(\Omega)^{2}$.

Здесь правая часть $f^{\varepsilon, h}$ зависит от $\varepsilon, h$, в то время как в (1.2) она была фиксированной. Предполагается, что последовательность $f^{\varepsilon, h}$ ограничена в $L^{2}\left(\Omega, d \mu_{\varepsilon}^{h}\right)^{N}$, т.e.

$$
\limsup _{\varepsilon \rightarrow 0} \int_{\Omega}\left|f^{\varepsilon, h}\right|^{2} d \mu_{\varepsilon}^{h}<\infty
$$

В интегральном тождестве (2.1) можно взять пробную функцию $\varphi=u^{\varepsilon, h}$ и получить энергетическое равенство

$$
\int_{\Omega}\left(A e\left(u^{\varepsilon, h}\right) \cdot e\left(u^{\varepsilon, h}\right)+u^{\varepsilon, h} \cdot u^{\varepsilon, h}\right) d \mu_{\varepsilon}^{h}=\int_{\Omega} f^{\varepsilon, h} \cdot u^{\varepsilon, h} d \mu_{\varepsilon}^{h}
$$

из которого следует, что

$$
u^{\varepsilon, h}, e\left(u^{\varepsilon, h}\right) \text { ограничены в } L^{2}\left(\Omega, d \mu_{\varepsilon}^{h}\right) .
$$

Видим, что необходимо изучать последовательности, ограниченные в “переменном" пространстве $L^{2}\left(\Omega, d \mu_{\varepsilon}^{h}\right)$. Специальньй математический аппарат позволяет это сделать: ограниченная последовательность оказывается компактной в смысле “слабой двухмасштабной сходимости”. Обшие свойства этой сходимости столь же просты, как и свойства классической слабой сходимости в $L^{2}(\Omega)$.

Наиболее интересные проблемы обнаруживаются при совместном рассмотрении вектор-функции $u^{\varepsilon, h}$ и ее тензора деформации $e\left(u^{\varepsilon, h}\right)$, когда выполнено условие (2.3). В этом случае слабые двухмасштабные пределы последовательностей $u^{\varepsilon, h}$ и $e\left(u^{\varepsilon, h}\right)$ связаны между собой и обладают важными для теории усреднения дополнительньми свойствами. Указанные вопросы рассматривались в работе [5], но были отмечены только такие свойства, которые справедливы при любом $h(\varepsilon) \rightarrow 0$.

В настоящей работе выясняется специфика критического случая. Значительная часть излагаемого материала не связана прямо с уравнениями, а относится к доказательству особых свойств слабого двухмасштабного предела произвольной последовательности $u^{\varepsilon, h} \in W_{\varepsilon, h}$, удовлетворяющей условию ограниченности (2.3). 
2.2. Далее мы приводим известные или очевидные факты, связанные с двухмасштабной сходимостью.

Напомним сначала понятие слабой сходимости в $L^{2}\left(\square, d \mu^{h}\right)$ и свойство среднего значения.

Если последовательность $a^{h}$ ограничена в $L^{2}\left(\square, d \mu^{h}\right)$, то слабая сходимость $a^{h} \rightarrow a$ в $L^{2}\left(\square, d \mu^{h}\right)$ означает, что

$$
a \in L^{2}(\square, d \mu), \quad \lim _{h \rightarrow 0} \int_{\square} a^{h} \psi d \mu^{h}=\int_{\square} a \psi d \mu \quad \forall \psi \in C_{\text {per }}^{\infty}(\square) .
$$

Свойство среднего значения. Пусть $\Omega$ - ограниченная измеримая по Жордану область и $a^{h} \rightarrow a$ в $L^{2}\left(\square, d \mu^{h}\right)$. Тогда $\forall \varphi \in C(\bar{\Omega})$

$$
\lim _{\varepsilon \rightarrow 0} \int_{\Omega} \varphi(x) a^{h}\left(\frac{x}{\varepsilon}\right) d \mu_{\varepsilon}^{h}=\int_{\Omega} \int_{\square} \varphi(x) a(y) d \mu .
$$

Фиксируем некоторую функцию $h(\varepsilon), h(\varepsilon) \rightarrow 0$ при $\varepsilon \rightarrow 0$.

ОПРЕДЕЛЕНИЕ 2.1. Ограниченная в $L^{2}\left(\Omega, d \mu_{\varepsilon}^{h}\right)$ последовательность $v^{\varepsilon, h}(x)$ слабо двухмасштабно сходится к функиии

$$
v=v(x, y) \in L^{2}(\Omega \times \square, d x \times d \mu)=L^{2}(\Omega \times \square), \quad v^{\varepsilon, h}(x) \stackrel{2}{\rightarrow} v(x, y),
$$

если

$$
\lim _{\varepsilon \rightarrow 0} \int_{\Omega} v^{\varepsilon, h}(x) \varphi(x) b\left(\frac{x}{\varepsilon}\right) d \mu_{\varepsilon}^{h}=\int_{\Omega} \int_{\square} v(x, y) \varphi(x) b(y) d x d \mu
$$

для любых $\varphi \in C_{0}^{\infty}(\Omega), b \in C_{\mathrm{per}}^{\infty}(\square)$.

Отметим некоторые свойства.

i) Ограниченная в $L^{2}\left(\Omega, d \mu_{\varepsilon}^{h}\right)$ последовательность компактна в смысле слабой двухмасштабной сходимости.

ii) Полунепрерывность снизу:

$$
\liminf _{\varepsilon \rightarrow 0} \int_{\Omega}\left|v^{\varepsilon, h}\right|^{2} d \mu_{\varepsilon}^{h} \geqslant \int_{\Omega} \int_{\square}|v|^{2} d x d \mu .
$$

ПРЕДЛОЖЕНИЕ 2.2. Если $c^{h} \rightarrow c$ в $L^{2}\left(\square, d \mu^{h}\right), m o c^{h}(x / \varepsilon) \stackrel{2}{\rightarrow} c(y)$.

ДокАЗАтЕльство. Рассмотрим выражение

$$
J(\varepsilon)=\int_{\Omega} c^{h}\left(\frac{x}{\varepsilon}\right) b\left(\frac{x}{\varepsilon}\right) \varphi(x) d \mu_{\varepsilon}^{h} .
$$

Так как $c^{h} b \rightarrow c b$ в $L^{2}\left(\square, d \mu^{h}\right)$, то по свойству среднего значения $(2.4)$ получаем

$$
\lim _{\varepsilon \rightarrow 0} J(\varepsilon)=\int_{\Omega} \int_{\square} c(y) b(y) \varphi(x) d x d \mu,
$$

что и требовалось.

В определении 2.1 используется достаточно узкий класс пробных функций $\varphi(x) b(y)$ (к ним автоматически добавляются и линейные комбинации). Но и его полезно сузить. 
ПРЕДЛОЖЕНИЕ 2.3. СХодимость $v^{\varepsilon, h} \stackrel{2}{a} v$ имеет место, если соотношение (2.5) выполнено для любих $\varphi \in C_{0}^{\infty}(\Omega)$ и любъх $b \in C_{\mathrm{per}}^{\infty}(\square)$, финитних в окрестности узлов сетки $F$.

Несложное доказательство опускается.

Узлами сетки $F^{h}$ (а также сжатой сетки $F_{\varepsilon}^{h}$ ) называем участки, принадлежащие двум или более $h$-стержням. Из предложения 2.3 получаем: если две ограниченные в $L^{2}\left(\Omega, d \mu_{\varepsilon}^{h}\right)$ последовательности отличаются только в узлах сетки $F_{\varepsilon}^{h}$, то они имеют одинаковые слабые двухмасштабные пределы.

Часто требуется расширить класс пробных функций, участвующих в определении 2.1, с сохранением самой сходимости (2.5). В частности, не только избавиться от гладкости функции $b$, но и сделать ее зависящей от $h$. Для этой цели привлечем понятие сильной сходимости в $L^{2}\left(\square, d \mu^{h}\right)$. Напомним, что ограниченная в $L^{2}\left(\square, d \mu^{h}\right)$ последовательность $b^{h}$ сильно сходится к функции $b \in L^{2}(\square, d \mu)$, $b^{h} \rightarrow b$, если

$$
\lim _{h \rightarrow 0} \int_{\square} b^{h} g^{h} d \mu^{h}=\int_{\square} b g d \mu, \quad \text { как только } g^{h} \rightarrow g \text { в } L^{2}\left(\square, d \mu^{h}\right) .
$$

Сильная сходимость складьвается из слабой сходимости и соотношения

$$
\lim _{h \rightarrow 0} \int_{\square}\left|b^{h}\right|^{2} d \mu^{h}=\int_{\square}|b|^{2} d \mu .
$$

Лемма 2.4. Пуств $v^{\varepsilon, h} \stackrel{2}{\rightarrow} v u b^{h} \rightarrow b$ в $L^{2}\left(\square, d \mu^{h}\right)$. Тогдa $\forall \varphi \in C(\bar{\Omega})$

$$
\lim _{\varepsilon \rightarrow 0} \int_{\Omega} v^{\varepsilon, h}(x) b^{h}\left(\frac{x}{\varepsilon}\right) \varphi(x) d \mu_{\varepsilon}^{h}=\int_{\Omega} \int_{\square} v(x, y) b(y) \varphi(x) d x d \mu .
$$

Мы подошли к понятию сильной двухмасштабной сходимости $u^{\varepsilon, h} \stackrel{2}{\rightarrow} u(x, y)$. По определению это означает, что

$$
\lim _{\varepsilon \rightarrow 0} \int_{\Omega} u^{\varepsilon, h}(x) z^{\varepsilon, h}(x) d \mu_{\varepsilon}^{h}=\int_{\Omega} \int_{\square} u(x, y) z(x, y) d x d \mu, \quad \text { как только } z^{\varepsilon, h} \stackrel{2}{-} z \text {. }
$$

Известно, что сильная двухмасштабная сходимость складывается из слабой и соотношения

$$
\lim _{\varepsilon \rightarrow 0} \int_{\Omega}\left|u^{\varepsilon, h}\right|^{2} d \mu_{\varepsilon}^{h}=\int_{\Omega} \int_{\square}|u|^{2} d x d \mu .
$$

ПРЕДЛОЖЕНИЕ 2.5. Если $c^{h} \rightarrow c$ в $L^{2}\left(\square, d \mu^{h}\right), \operatorname{mo~} c^{h}(x / \varepsilon) \stackrel{2}{\rightarrow} c(y)$.

Пример (естественное продолжение). Дана функция $g \in L^{2}(\square, d \mu)$. Продолжим эту функцию как постоянную в поперечном направлении на каждый $h$-стержень, а в узлах сетки $F^{h}$ определим ее как сумму таких продолжений. Получается функция $g_{h} \in L^{2}\left(\square, d \mu^{h}\right)$ и нетрудно убедиться, что $g_{h} \rightarrow g$ в $L^{2}\left(\square, d \mu^{h}\right)$. Таким образом, в силу предложения 2.5 для естественного продолжения имеем сходимость

$$
g_{h}\left(\frac{x}{\varepsilon}\right) \stackrel{2}{\rightarrow} g(y) .
$$

Понятие сильной двухмасштабной сходимости выглядит довольно громоздким, но при определенных условиях его можно упростить. 
ЛЕмма 2.6. Пусть $v^{\varepsilon, h}(x) \stackrel{2}{\rightarrow} v(x, y)$ и предельная функиия $v$ имеет струкmypy

$$
v(x, y)=\sum \varphi_{i}(x) b_{i}(y), \quad \varphi_{i} \in C(\bar{\Omega}), \quad b_{i} \in L^{2}(\square, d \mu)
$$

Тогда

$$
\lim _{\varepsilon \rightarrow 0} \int_{\Omega}\left|v^{\varepsilon, h}(x)-v_{h}\left(x, \frac{x}{\varepsilon}\right)\right|^{2} d \mu_{\varepsilon}^{h}=0
$$

где $v_{h}$ - это естественное продолжение функции $v(x, \cdot)$.

\section{§ 3. О двухмасштабной сходимости на периодической сетке критической толщины}

Здесь мы изучаем произвольную последовательность вектор-функций $u^{\varepsilon, h} \in$ $W_{\varepsilon, h}$, удовлетворяющую условию ограниченности (2.3). Не ограничивая общности, считаем ее слабо двухмасштабно сходящейся: $u^{\varepsilon, h}(x) \stackrel{2}{\rightarrow} u(x, y)$. Обшая теория, справедливая в случае произвольного $h(\varepsilon) \rightarrow 0$, дает некоторые сведения о структуре предельной функции, а именно,

$$
u^{\varepsilon, h}(x) \rightarrow u(x, y)=u^{0}(x)+\chi(x, y)
$$

где $u^{0} \in H_{0}^{1}(\Omega)^{2}, \chi \in L^{2}\left(\Omega, \mathscr{R}_{1}\right)$ (см. [5; теорема 12.3]).

Нашей целью будет следуюшее уточнение указанной структуры.

Теорема 3.1. В критическом случае $\chi \in L^{2}\left(\Omega, \mathscr{R}_{1}^{0}\right)$. Другими словами, предельная функиия $u(x, y)$ является әлементом әнергетического пространства усредненной задачи, т.е. $u \in V$.

Другое уточнение относится к свойству полунепрерывности снизу.

ТЕОРема 3.2. Пусть, дополнительно $\kappa(3.1)$, имеет место слабая двухмаситабная сходимость тензоров деформачии:

$$
e\left(u^{\varepsilon, h}(x)\right) \stackrel{2}{\rightarrow} p(x, y)
$$

Тогда справедливо неравенство

$$
\liminf _{\varepsilon \rightarrow 0} \int_{\Omega} A e\left(u^{\varepsilon, h}\right) \cdot e\left(u^{\varepsilon, h}\right) d \mu_{\varepsilon}^{h} \geqslant \int_{\Omega} \int_{\square}\left(A p \cdot p+\frac{\theta^{2}}{3} \rho \chi^{\prime \prime} \cdot \chi^{\prime \prime}\right) d x d \mu
$$

где $A$ - тензор упругости, $\rho$ - функиия, определенная равенством (1.20).

Отметим, что общая теория дает неравенство без второго слагаемого справа.

Приступим к доказательству теоремы 3.1 .

Напомним, что определение 1.1 пространства $\mathscr{R}_{1}^{0}$ включает условие гладкости на звенњях и условия сопряжения в узлах (см. условия а)-c) в $\S 1)$.

Ниже мы последовательно проверяем выполнение этих условий для функции $\chi$ из (3.1). 
3.1. Начнем с одной важной технической леммы. Пусть $I$ - отрезок, принадлежаший сетке $F$, причем безразлично, содержит он узел или нет. Можно считать, что он целиком лежит в ячейке периодичности.

Сначала рассмотрим случай горизонтального отрезка, расположенного на оси $O y_{1}$.

Введем 1-периодическую функцию, определенную на периоде $\left[-\frac{1}{2}, \frac{1}{2}\right]$ равенстBOM

$$
\beta_{0}(t)=\left\{\begin{array}{cc}
\frac{t}{h} & \text { при }|t| \leqslant h \\
0 & \text { при }|t|>h,
\end{array}\right.
$$

где $0<h \leqslant \frac{1}{8}$, и периодический вектор

$$
b(y)=b_{h}(y)=\left(\beta_{0}\left(y_{2}\right), 0\right)
$$

Изучим величину

$$
\begin{aligned}
J(\varepsilon) & =\frac{h}{\varepsilon^{2}} \int_{\Omega} u^{\varepsilon, h}(x) \cdot b\left(\frac{x}{\varepsilon}\right) \alpha\left(\frac{x_{1}}{\varepsilon}\right) \varphi(x) d \mu_{\varepsilon}^{h} \\
& =\frac{h}{\varepsilon^{2}} \int_{\Omega} u_{1}^{\varepsilon, h}(x) \beta_{0}\left(\frac{x_{2}}{\varepsilon}\right) \alpha\left(\frac{x_{1}}{\varepsilon}\right) \varphi(x) d \mu_{\varepsilon}^{h}
\end{aligned}
$$

где функция $\alpha\left(y_{1}\right) \in C_{0}^{\infty}(I)$ и периодически продолжена на $\mathbb{R}^{2}, \varphi \in C_{0}^{\infty}(\Omega)$.

ЛЕмма 3.3. Имеет место равенство

$$
\lim _{\varepsilon \rightarrow 0} J(\varepsilon)=\frac{\theta^{2}}{3} \int_{\Omega} \int_{\square \cap I} u_{2}(x, y) \alpha^{\prime}\left(y_{1}\right) \varphi(x) d x d \mu(y) .
$$

ДоказАтельство. Разобьем плоскость $\mathbb{R}^{2}$ на полуоткрытые квадраты $[0, \varepsilon)^{2}+\varepsilon n, n-$ целочисленный вектор, и обозначим отдельньй квадрат символом $\varepsilon \square_{j}$. Поскольку $\varphi \in C_{0}^{\infty}(\Omega)$, то для изучения величины $J(\varepsilon)$ достаточно ограничиться квадратами $\varepsilon \square_{j}$, целиком содержащимися в области $\Omega$. Их число имеет порядок $\varepsilon^{-2}$.

Интегрирование в (3.5) фактически ведется по системе периодических полосок вида $\varepsilon I^{h}+\varepsilon n$, где $I^{h}=I \times[-h, h]$. С функцией $\varphi(x) \in C_{0}^{\infty}(\Omega)$ свяжем функцию $\varphi_{\varepsilon}(x)$, определенную только на указанных полосках, причем так, что $\varphi_{\varepsilon}$ постоянна в поперечном направлении на каждой полоске и совпадает с $\varphi$ на средней линии. Очевидно, что

$$
\left|\varphi(x)-\varphi_{\varepsilon}(x)\right| \leqslant c \varepsilon^{2} .
$$

Отсюда следует, что в определении величины $J(\varepsilon)$ функцию $\varphi$ можно заменить на $\varphi_{\varepsilon}$, поскольку возникаюшая погрешность имеет порядок $O(\varepsilon)$ при $\varepsilon \rightarrow 0$. Действительно, пусть

$$
r(\varepsilon)=\frac{h}{\varepsilon^{2}} \int_{\Omega} u_{1}^{\varepsilon, h}(x) \beta_{0}\left(\frac{x}{\varepsilon}\right) \alpha\left(\frac{x_{1}}{\varepsilon}\right)\left(\varphi(x)-\varphi_{\varepsilon}(x)\right) d \mu_{\varepsilon}^{h} .
$$

Так как $\left|\beta_{0}\right| \leqslant 1$, то

$$
|r(\varepsilon)| \leqslant c h \int_{\Omega}\left|u_{1}^{\varepsilon, h}\right| d \mu_{\varepsilon}^{h}=O(\varepsilon) .
$$


Нам понадобятся некоторые преобразования для вектор-функции $v=v(y)=$ $v\left(y_{1}, y_{2}\right)$, заданной на полосе $I^{h}=I \times[-h, h]$. Исходим из равенства

$$
\int_{-h}^{h} y_{2} v_{1} d y_{2}=\frac{h^{2}}{2} \int_{-h}^{h} \frac{\partial v_{1}}{\partial y_{2}} d y_{2}-\frac{1}{2} \int_{-h}^{h} y_{2}^{2} \frac{\partial v_{1}}{\partial y_{2}} d y_{2}
$$

получающегося интегрированием по частям. Так как

$$
\frac{1}{2} \frac{\partial v_{1}}{\partial y_{2}}=e_{12}(v)-\frac{1}{2} \frac{\partial v_{2}}{\partial y_{1}}
$$

TO

$$
\int_{-h}^{h} y_{2} v_{1} d y_{2}=\frac{1}{2} \int_{-h}^{h}\left(y_{2}^{2}-h^{2}\right) \frac{\partial v_{2}}{\partial y_{1}} d y_{2}+\int_{-h}^{h} e_{12}(v)\left(h^{2}-y_{2}^{2}\right) d y_{2} .
$$

Отсюда для $g \in C_{0}^{\infty}(I)$ имеем

$$
\int_{I^{h}} y_{2} g\left(y_{1}\right) v_{1}(y) d y=\frac{1}{2} \int_{I^{h}}\left(y_{2}^{2}-h^{2}\right) g\left(y_{1}\right) \frac{\partial v_{2}}{\partial y_{1}} d y+\int_{I^{h}} e_{12}(v) g\left(y_{1}\right)\left(h^{2}-y_{2}^{2}\right) d y .
$$

Преобразуем первое слагаемое справа. Интегрируя по частям по переменной $y_{1}$ и полагая

$$
\bar{v}_{2}\left(y_{1}\right)=\frac{1}{2 h} \int_{-h}^{h} v_{2}\left(y_{1}, y_{2}\right) d y_{2}
$$

получаем

$$
\begin{aligned}
\frac{1}{2} \int_{I^{h}}\left(h^{2}-y_{2}^{2}\right) v_{2}(y) g^{\prime}\left(y_{1}\right) d y= & \frac{1}{2} \int_{I^{h}}\left(h^{2}-y_{2}^{2}\right) \bar{v}_{2}\left(y_{1}\right) g^{\prime}\left(y_{1}\right) d y \\
& +\frac{1}{2} \int_{I^{h}}\left(h^{2}-y_{2}^{2}\right)\left(v_{2}-\bar{v}_{2}\right) g^{\prime}\left(y_{1}\right) d y \\
\frac{1}{2} \int_{I^{h}}\left(h^{2}-y_{2}^{2}\right) \bar{v}_{2}\left(y_{1}\right) g^{\prime}\left(y_{1}\right) d y= & \frac{2}{3} h^{3} \int_{I} \bar{v}_{2} g^{\prime} d y_{1}=\frac{1}{3} h^{2} \int_{I^{h}} v_{2} g^{\prime} d y .
\end{aligned}
$$

Итак, справедливо равенство

$$
\begin{aligned}
\int_{I^{h}} y_{2} v_{1}(y) g\left(y_{1}\right) d y-\frac{1}{3} h^{2} \int_{I^{h}} v_{2}(y) g^{\prime}\left(y_{1}\right) d y \\
=\frac{h^{2}}{2} \int_{I^{h}} g(y)\left(\frac{\partial v_{1}}{\partial y_{2}}+\frac{\partial v_{2}}{\partial y_{1}}\right)\left(1-\left(\frac{y_{2}}{h}\right)^{2}\right) d y \\
+\frac{h^{2}}{2} \int_{I^{h}}\left(v_{2}-\bar{v}_{2}\right) g^{\prime}\left(y_{1}\right)\left(1-\left(\frac{y_{2}}{h}\right)^{2}\right) d y
\end{aligned}
$$

Для вектора $v=v(x)$, заданного в полосе $\varepsilon I^{h}$, соответствующее равенство получается гомотетией и имеет вид

$$
\begin{aligned}
\frac{h}{\varepsilon^{2}} \int_{\varepsilon I^{h}} \beta_{0}\left(\frac{x_{2}}{\varepsilon}\right) v_{1}(x) g\left(\frac{x_{1}}{\varepsilon}\right) d x-\frac{1}{3}\left(\frac{h}{\varepsilon}\right)^{2} \int_{\varepsilon I^{h}} v_{2}(x) g^{\prime}\left(\frac{x_{1}}{\varepsilon}\right) d x \\
=\frac{1}{2} \frac{h^{2}}{\varepsilon} \int_{\varepsilon I^{h}}\left(\frac{\partial v_{1}}{\partial x_{2}}+\frac{\partial v_{2}}{\partial x_{1}}\right) g\left(\frac{x_{1}}{\varepsilon}\right)\left(1-\left(\beta_{0}\left(\frac{x_{2}}{\varepsilon}\right)\right)^{2}\right) d x \\
\quad+\frac{1}{2}\left(\frac{h}{\varepsilon}\right)^{2} \int_{\varepsilon I^{h}}\left(v_{2}(x)-\bar{v}_{2}\left(x_{1}\right)\right) g^{\prime}\left(\frac{x_{1}}{\varepsilon}\right)\left(1-\left(\beta_{0}\left(\frac{x_{2}}{\varepsilon}\right)\right)^{2}\right) d x=T_{1}+T_{2}
\end{aligned}
$$


Оценим стоящие справа слагаемые. Очевидно, что

$$
\left|T_{1}\right| \leqslant \frac{l h^{2}}{\varepsilon} \int_{\varepsilon I^{h}}|e(v)| d x
$$

где $l=\max \left(|g|+\left|g^{\prime}\right|\right)$. Для второго слагаемого с помощью неравенства Пуанкаре

$$
\int_{\varepsilon I_{h}}\left|v_{2}-\bar{v}_{2}\right| d x \leqslant c_{0} \varepsilon h \int_{\varepsilon I^{h}}\left|\frac{\partial v_{2}}{\partial x_{2}}\right| d x \leqslant c_{0} h \varepsilon \int_{\varepsilon I^{h}}|e(v)| d x
$$

получим аналогичную оценку

$$
\left|T_{2}\right| \leqslant \frac{c_{0} l h^{2}}{\varepsilon} \int_{\varepsilon I^{h}}|e(v)| d x .
$$

Перепишем эти результаты в терминах меры $\mu_{\varepsilon}^{h}$. Пусть $\rho$-характеристическая функция полосы $I^{h}$, периодически продолженная на $\mathbb{R}^{2}$. Тогда выполнена оценка

$$
\begin{gathered}
\left|\frac{h}{\varepsilon^{2}} \int_{\varepsilon \square} \beta_{0}\left(\frac{x_{2}}{\varepsilon}\right) v_{1}(x) g\left(\frac{x_{1}}{\varepsilon}\right) d \mu_{\varepsilon}^{h}-\frac{1}{3}\left(\frac{h}{\varepsilon}\right)^{2} \int_{\varepsilon \square} v_{2}(x) g^{\prime}\left(\frac{x_{1}}{\varepsilon}\right) \rho\left(\frac{x}{\varepsilon}\right) d \mu_{\varepsilon}^{h}\right| \\
\leqslant \frac{c_{1} l h^{2}}{\varepsilon} \int_{\varepsilon \square}|e(v)| d \mu_{\varepsilon}^{h} .
\end{gathered}
$$

Теперь вернемся к величине $J(\varepsilon)$.

Используем оценку (3.7) в каждом квадрате $\varepsilon \square_{j}$, полагая в ней

$$
v(x)=u^{\varepsilon, h}(x), \quad g\left(y_{1}\right)=\alpha\left(y_{1}\right) \varphi_{\varepsilon}\left(\varepsilon y_{1}\right)
$$

Поскольку в данном случае

$$
g^{\prime}\left(\frac{x}{\varepsilon}\right)=\alpha^{\prime}\left(\frac{x}{\varepsilon}\right) \varphi_{\varepsilon}(x)+O(\varepsilon)=\alpha^{\prime}\left(\frac{x}{\varepsilon}\right) \varphi(x)+O(\varepsilon),
$$

Tо

$$
\begin{gathered}
J(\varepsilon)=\frac{1}{3}\left(\frac{h}{\varepsilon}\right)^{2} \int_{\Omega} u_{2}^{\varepsilon, h}(x) \alpha^{\prime}\left(\frac{x_{1}}{\varepsilon}\right) \varphi(x) \rho\left(\frac{x}{\varepsilon}\right) d \mu_{\varepsilon}^{h}+r(\varepsilon), \\
|r(\varepsilon)| \leqslant c h^{2} \varepsilon^{-1} \int_{\Omega}\left(\left|u^{\varepsilon, h}\right|+\left|e\left(u^{\varepsilon, h}\right)\right|\right) d \mu_{\varepsilon}^{h}=O(\varepsilon) .
\end{gathered}
$$

Отсюда следует равенство (3.6) и лемма доказана. 
Сформулируем аналог леммы 3.3 в случае произвольно направленного отрезка. Пусть $\tau$ - направление вдоль отрезка $I, \nu$ - нормаль к нему, $\tau, \nu$ - правая пара. Через $I^{h}$ обозначим стержень (полосу) ширины $2 h$ со средней линией $I$.

На ячейке $\square$ определим функцию

$$
\beta(y)= \begin{cases}\frac{1}{h} \nu \cdot\left(y-y_{0}\right) & \text { на } I^{h} ; \\ 0 & \text { на } \square \backslash I^{h},\end{cases}
$$

где $y_{0} \in I$, и периодически продолжим ее на $\mathbb{R}^{2}$. Функция $\beta$ совпадает с $\beta_{0}$ (см. (3.4)) в случае горизонтального отрезка, лежащего на оси $O y_{1}$. Пусть $\alpha(y)-$ гладкая функция, зависящая только от продольной компоненты на стержне $I^{h}$ и равная нулю в окрестности его торцов. Тогда выполнено равенство

$$
\begin{aligned}
\lim _{\varepsilon \rightarrow 0} & \frac{h}{\varepsilon^{2}} \int_{\Omega} u^{\varepsilon, h}(x) \cdot \tau \beta\left(\frac{x}{\varepsilon}\right) \alpha\left(\frac{x}{\varepsilon}\right) \varphi(x) d \mu_{\varepsilon}^{h} \\
& =\frac{\theta^{2}}{3} \int_{\Omega} \int_{\square \cap I} u(x, y) \cdot \nu \frac{\partial \alpha(y)}{\partial \tau}(y) \varphi(x) d x d \mu(y), \quad \varphi \in C_{0}^{\infty}(\Omega) .
\end{aligned}
$$

В случае вертикального отрезка, лежащего на оси $O y_{2}$, имеем $\tau=(0,1), \nu=$ $(-1,0)$

$$
\begin{aligned}
\lim _{\varepsilon \rightarrow 0} & \frac{h}{\varepsilon^{2}} \int_{\Omega} u_{2}^{\varepsilon, h}(x) \beta_{0}\left(\frac{x_{1}}{\varepsilon}\right) \alpha\left(\frac{x_{2}}{\varepsilon}\right) \varphi(x) d \mu_{\varepsilon}^{h} \\
= & \frac{\theta^{2}}{3} \int_{\Omega} \int_{\square \cap I} u_{1}(x, y) \alpha^{\prime}\left(y_{2}\right) \varphi(x) d x d \mu(y) .
\end{aligned}
$$

Теперь мы в состоянии доказать следующую лемму.

ЛЕмма 3.4. На каждом отрезке I, принадлежсащем сетке $F$, нормальная компонента $u \cdot \nu$ есть әлемент пространства $H^{2}(I)$.

ДокАЗАТЕЛьство. Начнем со случая горизонтального отрезка и рассмотрим выражение

$$
T(\varepsilon)=-\frac{h}{\varepsilon} \int_{\Omega} \frac{\partial u_{1}^{\varepsilon, h}}{\partial x_{1}}(x) \beta_{0}\left(\frac{x_{2}}{\varepsilon}\right) \alpha\left(\frac{x_{1}}{\varepsilon}\right) \varphi(x) d \mu_{\varepsilon}^{h} .
$$

После интегрирования в нем по частям получим

$$
\begin{aligned}
T(\varepsilon)= & \frac{h}{\varepsilon^{2}} \int_{\Omega} u_{1}^{\varepsilon, h}(x) \beta_{0}\left(\frac{x_{2}}{\varepsilon}\right) \alpha^{\prime}\left(\frac{x_{1}}{\varepsilon}\right) \varphi(x) d \mu_{\varepsilon}^{h} \\
& +\frac{h}{\varepsilon} \int_{\Omega} u_{1}^{\varepsilon, h}(x) \beta_{0}\left(\frac{x_{2}}{\varepsilon}\right) \alpha\left(\frac{x_{1}}{\varepsilon}\right) \frac{\partial \varphi(x)}{\partial x_{1}} d \mu_{\varepsilon}^{h} .
\end{aligned}
$$

Первое слагаемое имеет тип $J(\varepsilon)($ см. $(3.5))$, а второе - тип $\varepsilon J(\varepsilon)$. По лемме 3.3

$$
\lim _{\varepsilon \rightarrow 0} T(\varepsilon)=\frac{\theta^{2}}{3} \int_{\Omega} \int_{\square \cap I} u_{2}(x, y) \alpha^{\prime \prime}\left(y_{1}\right) \varphi(x) d x d \mu .
$$


Изучим величину $T(\varepsilon)$ иным способом. Так как $\left|\beta_{0}\right| \leqslant 1$, то последовательность

$$
w_{\varepsilon}(x)=-\frac{\partial u_{1}^{\varepsilon, h}(x)}{\partial x_{1}} \beta_{0}\left(\frac{x_{2}}{\varepsilon}\right) \text { ограничена в } L^{2}\left(\Omega, d \mu_{\varepsilon}^{h}\right) .
$$

Переходя, если требуется, к подпоследовательности, считаем ее слабо двухмасштабно сходящейся, $w_{\varepsilon}(x) \stackrel{2}{\rightarrow} w(x, y), w \in L^{2}(\Omega \times \square)$. В частности,

$$
\lim _{\varepsilon \rightarrow 0} T(\varepsilon)=\lim _{\varepsilon \rightarrow 0} \int_{\Omega} w_{\varepsilon}(x) \alpha\left(\frac{x_{1}}{\varepsilon}\right) \varphi(x) d \mu_{\varepsilon}^{h}=\int_{\Omega} \int_{\square \cap I} w(x, y) \alpha\left(y_{1}\right) \varphi(x) d x d \mu(y) .
$$

Сравним это соотношение с (3.10). Поскольку $\varphi \in C_{0}^{\infty}(\Omega)$ произвольно, то для п.в. $x \in \Omega$ вьполнено равенство

$$
\int_{I} w(x, y) \alpha\left(y_{1}\right) d y_{1}=\frac{\theta^{2}}{3} \int_{I}\left(u_{2}^{0}(x)+\chi_{2}(x, y)\right) \alpha^{\prime \prime}\left(y_{1}\right) d y_{1},
$$

из которого следует, что

$$
\left.w(x, \cdot)\right|_{I}=\left.\frac{\theta^{2}}{3} \frac{\partial^{2} \chi_{2}}{\partial y_{1}^{2}}\right|_{I}
$$

или

$$
\left.\frac{\theta^{2}}{3}(\chi \cdot \nu)_{\tau \tau}^{\prime \prime}\right|_{I}=\left.w(x, \cdot)\right|_{I}, \quad w \in L^{2}(\Omega \times I)
$$

где $\nu=(0,1), \tau=(1,0)$.

Для отрезка общего положения рассуждения аналогичны. Рассматриваем выражение

$$
T(\varepsilon)=-\frac{h}{\varepsilon} \int_{\Omega} e\left(u^{\varepsilon, h}\right) \cdot(\tau \times \tau) \beta\left(\frac{x}{\varepsilon}\right) \alpha\left(\frac{x}{\varepsilon}\right) \varphi(x) d \mu_{\varepsilon}^{h},
$$

где $\beta(y), \alpha(y)$ - те же, что и в (3.8). Интегрируем в этом выражении по частям, используя равенство

$$
e(v) \cdot(\tau \times \tau)=\frac{d}{d \tau}(v \cdot \tau)
$$

Тогда получаем, что

$$
\lim _{\varepsilon \rightarrow 0} T(\varepsilon)=\frac{\theta^{2}}{3} \int_{\Omega} \int_{\square \cap I} u \cdot \nu \frac{\partial^{2} \alpha(y)}{\partial \tau^{2}} \varphi(x) d x d \mu(y) .
$$

Отсюда делаем вывод, что и в случае отрезка обшего положения выполнено соотношение (3.11), в котором $w$ есть слабый двухмасштабный предел последовательности

$$
-\frac{h}{\varepsilon} e\left(u^{\varepsilon, h}\right) \cdot(\tau \times \tau) \beta\left(\frac{x}{\varepsilon}\right) .
$$

Лемма доказана.

На самом деле, по ходу доказательства леммы 3.4 была установлена важная для дальнейшего сходимость. Выделим этот результат в отдельную лемму.

Рассмотрим всю сингулярную сетку, выбрав на каждом звене точку $y_{0}$ и направления $\tau$ и $\nu$ - тангенциальное и нормальное. Определим на сетке $F^{h}$ матрицу $\sigma(y)$,

$$
\sigma(y)=-(\tau \times \tau) \beta(y), \quad \text { где } \beta(y)=\frac{\nu \cdot\left(y-y_{0}\right)}{h} \text { на } h \text {-стержне. }
$$

В узлах сетки $F^{h}$ функцию $\beta$ полагаем равной нулю. 
ЛЕмма 3.5. Имеет место слабая двухмасштабная сходимость

$$
\frac{h}{\varepsilon} e\left(u^{\varepsilon, h}\right) \cdot \sigma\left(\frac{x}{\varepsilon}\right) \stackrel{2}{\rightarrow} \frac{\theta^{2}}{3}(\chi \cdot \nu)_{\tau \tau}^{\prime \prime}
$$

3.2. Приступим к выяснению условий сопряжения в узлах, а именно, к доказательству равенства (1.11) для первых производных.

Достаточно рассмотреть два сходящихся в узле звена и для них установить соответствующее равенство. Чтобы избежать громоздких обозначений, рассмотрим два перпендикулярных отрезка $I_{1}, I_{2}$, исходящих из начала координат в направлении координатных осей. Длину каждого из них считаем равной $\frac{1}{4}$. На горизонтальном отрезке $I_{1}$ функцию $u$ записываем как $u\left(x, y_{1}\right)$, а на вертикальном как $u\left(x, y_{2}\right)$. Условие $(1.11)$ равенства производных имеет вид

$$
\frac{\partial u_{2}}{\partial y_{1}}(x, 0)+\frac{\partial u_{1}}{\partial y_{2}}(x, 0)=0
$$

Идея доказательства равенства (3.14) состоит в следующем. С ломаной $I_{1} \cup I_{2}$ свяжем “ломаную" полосу, составленную из двух полос - горизонтальной полосы $I_{1}^{h}$ и вертикальной полосы $I_{2}^{h}, P=P^{h}=I_{1}^{h} \cup I_{2}^{h}$ (см. рис. 3 ).
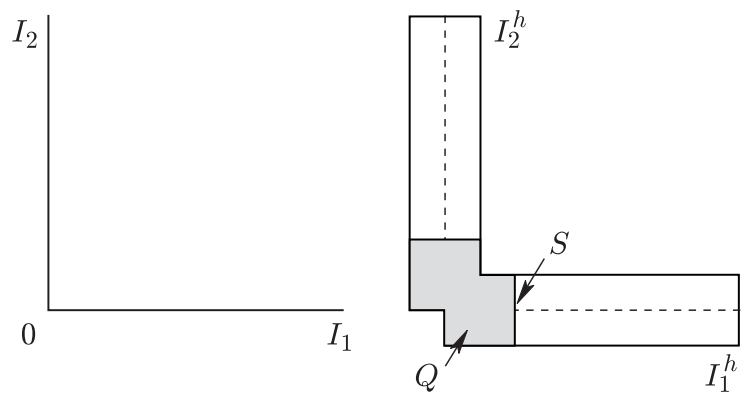

Рис. 3. Область $P$

На ячейке периодичности $\square$ зададим вектор $b$ равенством

$$
b(y)=b^{h}(y)= \begin{cases}\frac{1}{h}\left(y_{2} \Phi^{\prime}\left(y_{1}\right), 0\right) & \text { на } I_{1}^{h} ; \\ \frac{1}{h}\left(0, y_{1} \Phi^{\prime}\left(y_{2}\right)\right) & \text { на } I_{2}^{h} ; \\ 0 & \text { на } \square \backslash P^{h}\end{cases}
$$

и периодически продолжим его на $\mathbb{R}^{2}$. Здесь $\Phi^{\prime} \in C_{0}^{\infty}\left(0, \frac{1}{4}\right)$, а первообразная $\Phi$ нормирована условием $\Phi\left(\frac{1}{4}\right)=0$. В частности, вблизи узла вектор $b$ равен 0 (если только $h$ достаточно мало). Введем выражение

$$
l(\varepsilon)=\frac{h}{\varepsilon^{2}} \int_{\Omega} u^{\varepsilon, h} \cdot b\left(\frac{x}{\varepsilon}\right) \varphi(x) d \mu_{\varepsilon}^{h}, \quad \varphi \in C_{0}^{\infty}(\Omega)
$$


которое благодаря структуре вектора $b$ имеет вид

$$
\begin{aligned}
l(\varepsilon)= & \frac{h}{\varepsilon^{2}} \int_{\Omega} u_{1}^{\varepsilon, h}(x) \beta_{0}\left(\frac{x_{2}}{\varepsilon}\right) \Phi^{\prime}\left(\frac{x_{1}}{\varepsilon}\right) \varphi(x) d \mu_{\varepsilon}^{h} \\
& +\frac{h}{\varepsilon^{2}} \int_{\Omega} u_{2}^{\varepsilon, h}(x) \beta_{0}\left(\frac{x_{1}}{\varepsilon}\right) \Phi^{\prime}\left(\frac{x_{2}}{\varepsilon}\right) \varphi(x) d \mu_{\varepsilon}^{h}
\end{aligned}
$$

Из соотношений (3.6), (3.9) следует равенство

$$
\begin{aligned}
l= & \lim _{\varepsilon \rightarrow 0} l(\varepsilon)=\frac{\theta^{2}}{3} \int_{\Omega} \int_{\square \cap I_{1}} \varphi(x) u_{2}(x, y) \Phi^{\prime \prime}\left(y_{1}\right) d x d \mu(y) \\
& +\frac{\theta^{2}}{3} \int_{\Omega} \int_{\square \cap I_{2}} \varphi(x) u_{1}(x, y) \Phi^{\prime \prime}\left(y_{2}\right) d x d \mu(y) .
\end{aligned}
$$

Пока ничего нового не получено - мы просто сложили соотношения (3.6) и (3.9).

$\mathrm{C}$ другой стороны, величина $l=\lim _{\varepsilon \rightarrow 0} l(\varepsilon)$ может быть изучена другим способом, что приводит к следующему результату.

ПРЕДЛОЖЕНИЕ 3.6. Рассмотрим величину $l$ как линейный функиионал от $\Phi$ при фиксированном $\varphi \in C_{0}^{\infty}(\Omega)$. Тогда справедливо неравенство

$$
|l|^{2} \leqslant c \int_{0}^{1 / 4}|\Phi|^{2} d t, \quad c=c(\varphi)
$$

Воспользуемся этим неравенством. Имеем

$$
\frac{3}{\theta^{2}} l=\int_{\Omega} \varphi(x)\left[\int_{0}^{1 / 4} u_{2}\left(x, y_{1}\right) \Phi^{\prime \prime}\left(y_{1}\right) d y_{1}+\int_{0}^{1 / 4} u_{1}\left(x, y_{2}\right) \Phi^{\prime \prime}\left(y_{2}\right) d y_{2}\right] d x
$$

В квадратных скобках дважды произведем интегрирование по частям. Учитывая, что $\Phi^{\prime} \in C_{0}^{\infty}\left(0, \frac{1}{4}\right)$, получаем

$$
\begin{aligned}
\frac{3}{\theta^{2}} l= & \Phi(0) \int_{\Omega}\left(\frac{\partial u_{2}}{\partial y_{1}}(x, 0)+\frac{\partial u_{1}}{\partial y_{2}}(x, 0)\right) \varphi(x) d x \\
& +\int_{\Omega} \varphi(x)\left(\int_{0}^{1 / 4} \frac{\partial^{2} u_{2}}{\partial y_{1}^{2}}\left(x, y_{1}\right) \Phi\left(y_{1}\right) d y_{1}+\int_{0}^{1 / 4} \frac{\partial^{2} u_{1}}{\partial y_{2}^{2}}\left(x, y_{2}\right) \Phi\left(y_{2}\right) d y_{2}\right) d x
\end{aligned}
$$

Второе слагаемое непрерьвно по норме $\left(\int_{0}^{1 / 4}|\Phi|^{2} d t\right)^{1 / 2}$ в силу (3.11), а для непрерывности первого слагаемого требуется, чтобы

$$
\int_{\Omega}\left(\frac{\partial u_{2}}{\partial y_{1}}(x, 0)+\frac{\partial u_{1}}{\partial y_{2}}(x, 0)\right) \varphi(x) d x=0 \quad \forall \varphi \in C_{0}^{\infty}(\Omega)
$$

Отсюда следует искомое равенство (3.14). 
3.3. ДОКАЗАТЕЛЬСТВО ПРЕДЛОЖЕНИЯ 3.6. В области $P$ (см. рис. 3 ) рассмотрим задачу Неймана для уравнения $-\operatorname{div}(e(v))=b$, где вектор $b$ задан равенством (3.15). По определению ее решение - это функция

$$
v \in H^{1}(P)^{2}, \quad \int_{P} e(v) \cdot e(\varphi) d y=\int_{P} b \cdot \varphi d y \quad \forall \varphi \in C^{\infty}(\bar{P})^{2} .
$$

Для разрешимости этой задачи требуется, чтобы вектор $b$ был ортогонален на $P$ всем жестким перемешениям, т.е. векторам вида $c+t\left(-y_{2}, y_{1}\right)$. Выполнение этого условия ортогональности следует из структуры вектора $b$ (см. (3.15)). Решение $v$ определено с точностью до жесткого перемещения (ниже мы этим воспользуемся).

Проверим оценку

$$
\lim _{h \rightarrow 0} \int_{P} e(v) \cdot e(v) d \mu^{h} \leqslant c \int_{0}^{1 / 4}|\Phi|^{2} d t .
$$

В тождестве (3.17) возьмем пробную функцию $\varphi=v$. Тогда получим

$$
h \int_{\square} e(v) \cdot e(v) d y=\int_{I_{1}^{h}} y_{2} \Phi^{\prime}\left(y_{1}\right) v_{1}(y) d y+\int_{I_{2}^{h}} y_{1} \Phi^{\prime}\left(y_{2}\right) v_{2}(y) d y=T_{1}+T_{2} .
$$

Оценим величину $T_{1}$. Рассмотрим заштрихованный на рис. 3 многоугольник $Q$, составленный из двух квадратов со стороной $2 h$, и пусть $S$ - правая сторона квадрата, принадлежашего полосе $I_{1}^{h}$. Напомним, что функция $\Phi^{\prime}\left(y_{1}\right)$ равна нулю в $Q$.

Из (3.19) в результате интегрирования по частям имеем

$$
\begin{aligned}
T_{1} & =-\int_{I_{1}^{h}} y_{2} \Phi \frac{\partial v_{1}}{\partial y_{1}} d y-\int_{S} \Phi(0) y_{2} v_{1} d y_{2}=T_{1}^{\prime}+T_{1}^{\prime \prime} \\
\left|T_{1}^{\prime}\right| & \leqslant 2 h^{3 / 2}\left(\int_{0}^{1 / 4}|\Phi|^{2} d t\right)^{1 / 2}\left(\int_{P} e(v) \cdot e(v) d y\right)^{1 / 2}, \\
\left|T_{1}^{\prime \prime}\right| & \leqslant|\Phi(0)| h^{3 / 2}\left(\int_{S}\left|v_{1}\right|^{2} d y_{2}\right)^{1 / 2} \leqslant c|\Phi(0)| h^{2}\left(\int_{Q} e(v) \cdot e(v) d y\right)^{1 / 2} .
\end{aligned}
$$

Во второй оценке на последнем шаге использовано неравенство

$$
\int_{S}\left|v_{1}\right|^{2} d y_{2} \leqslant c h \int_{Q} e(v) \cdot e(v) d y
$$

которое доказьвается следуюшим образом. По теореме о следе

$$
\int_{S}\left|v_{1}\right|^{2} d y_{2} \leqslant c\left(h \int_{Q}\left|\nabla v_{1}\right|^{2} d y+h^{-1} \int_{Q}\left|v_{1}\right|^{2} d y\right) .
$$

Теперь нужно решение $v$ выбрать ортогональным на $Q$ всем жестким перемешениям и воспользоваться неравенством Корна

$$
h^{-2} \int_{Q}|v|^{2} d y+\int_{Q}|\nabla v|^{2} d y \leqslant c \int_{Q} e(v) \cdot e(v) d y .
$$


Мы оценили величину $T_{1}$ из (3.19). Оценивая величину $T_{2}$ аналогичным способом, получаем неравенство

$h \int_{P} e(v) \cdot e(v) d y \leqslant c\left(\int_{P} e(v) \cdot e(v) d y\right)^{1 / 2}\left(h^{3 / 2}\left(\int_{0}^{1 / 4}|\Phi|^{2} d t\right)^{1 / 2}+c_{1} h^{2}|\Phi(0)|\right)$

из которого следует искомое неравенство (3.18), если учесть, что $d \mu^{h}(y)=(c / h) d y$, $c>0$.

Далее положим

$$
g^{h}(y)= \begin{cases}e(v) & \text { на } P \\ 0 & \text { на } \square \backslash P\end{cases}
$$

и продолжим $g^{h}$ периодически на $\mathbb{R}^{2}$. Тогда оценка $(3.18)$ перепишется в виде

$$
\lim _{h \rightarrow 0} \int_{\square}\left|g^{h}\right|^{2} d \mu^{h} \leqslant c \int_{0}^{1 / 4}|\Phi|^{2} d t
$$

а из тождества (3.17) следует, что

$$
\varepsilon \int_{\Omega} g^{h}\left(\frac{x}{\varepsilon}\right) \cdot e(\psi(x)) d x=\int_{\Omega} b^{h}\left(\frac{x}{\varepsilon}\right) \cdot \psi(x) d x, \quad \psi \in C_{0}^{\infty}(\Omega)^{2} .
$$

Поскольку $g^{h}(x / \varepsilon)$ и $b^{h}(x / \varepsilon)$ сосредоточены на сетке $F_{\varepsilon}^{h}$, то вместо $d x$ можно взять $d \mu_{\varepsilon}^{h}$, т.е.

$$
\varepsilon \int_{\Omega} g^{h}\left(\frac{x}{\varepsilon}\right) \cdot e(\psi(x)) d \mu_{\varepsilon}^{h}=\int_{\Omega} b^{h}\left(\frac{x}{\varepsilon}\right) \cdot \psi(x) d \mu_{\varepsilon}^{h}, \quad \psi \in C_{0}^{\infty}(\Omega)^{2} .
$$

Полагая в этом тождестве $\psi=\varphi(x) u^{\varepsilon, h}(x)$, где $\varphi \in C_{0}^{\infty}(\Omega)$, получаем

$$
\int_{\Omega} g^{h} \cdot e\left(\varphi u^{\varepsilon, h}\right) d \mu_{\varepsilon}^{h}=\frac{1}{\varepsilon} \int_{\Omega} u^{\varepsilon, h}(x) \cdot b^{h}\left(\frac{x}{\varepsilon}\right) \varphi(x) d \mu_{\varepsilon}^{h}=\frac{\varepsilon}{h} l(\varepsilon) .
$$

Последовательность $e\left(\varphi u^{\varepsilon, h}\right)$ ограничена в $L^{2}\left(\Omega, d \mu_{\varepsilon}^{h}\right)$ и по неравенству Коши-Буняковского

$$
|l(\varepsilon)|^{2} \leqslant c\left(\frac{h}{\varepsilon}\right)^{2} \int_{\Omega}\left|g^{h}\left(\frac{x}{\varepsilon}\right)\right|^{2} d \mu_{\varepsilon}^{h}, \quad c=c(\varphi) .
$$

Покроем область $\Omega$ квадратами $\varepsilon \square_{j}$ (их число имеет порядок $O\left(\varepsilon^{-2}\right)$ ). Тогда

$$
\begin{aligned}
\int_{\Omega}\left|g^{h}\left(\frac{x}{\varepsilon}\right)\right|^{2} d \mu_{\varepsilon}^{h} & \leqslant \sum \int_{\varepsilon \square_{j}}\left|g^{h}\left(\frac{x}{\varepsilon}\right)\right|^{2} d \mu_{\varepsilon}^{h}=\sum \varepsilon^{2} \int_{\square}\left|g^{h}(y)\right|^{2} d \mu^{h} \\
& \leqslant 2|\Omega| \int_{\square}\left|g^{h}\right|^{2} d \mu^{h} .
\end{aligned}
$$

Отсюда и из (3.20) следует искомое неравенство (3.16).

3.4. Займемся проверкой условия закрепления в узлах. Будем опираться на следуюшую теорему из теории двухмасштабной сходимости. В ней речь идет о скалярных функциях, причем на структуру сетки накладываются весьма слабые ограничения. 
Теорема 3.7. Допустим, что сингулярная сетка F удовлетворяет единственному условию - она связна на торе периодичности. Пусть далее $h(\varepsilon) \rightarrow 0$ произвольно и последовательность гладких скалярных функиий такова, что

$$
\varepsilon^{2} \int_{\Omega}\left|\nabla v^{\varepsilon, h}\right|^{2} d \mu_{\varepsilon}^{h}+\int_{\Omega}\left|v^{\varepsilon, h}\right|^{2} d \mu_{\varepsilon}^{h} \leqslant c_{1}<\infty .
$$

Тогда (с точностью до въделения подпоследовательности)

$$
\begin{gathered}
v^{\varepsilon, h}(x) \stackrel{2}{\rightarrow} v(x, y), \quad v(x, \cdot) \in H_{\mathrm{per}}^{1}(\square, d \mu) \quad \text { для n.в. } \quad x \in \Omega, \\
\varepsilon \nabla v^{\varepsilon, h}(x) \stackrel{2}{\longrightarrow} \nabla_{y} v(x, y),
\end{gathered}
$$

где $H_{\mathrm{per}}^{1}(\square, d \mu)$ - соболевское пространство периодических функиий.

Более подробно эта теорема обсуждается в $\S 5$.

Для применения теоремы 3.7 нужна оценка вида (3.21) для каждой компоненты вектора $u^{\varepsilon, h}$. Такая оценка получается из следующего неравенства типа неравенства Корна

$$
h^{2} \int_{\Omega}|\nabla u|^{2} d \mu_{\varepsilon}^{h} \leqslant c \int_{\Omega}\left(e(u) \cdot e(u)+\left(\frac{h}{\varepsilon}\right)^{2} u \cdot u\right) d \mu_{\varepsilon}^{h}, \quad u \in C_{0}(\Omega)^{2},
$$

которое будет доказано в $\S 5$. В критическом случае это неравенство обеспечивает оценку вида (3.21) для каждой компоненты вектора $u^{\varepsilon, h}$.

Таким образом, по теореме 3.7 каждая компонента вектора $\chi$ принадлежит (для п.в. $x \in \Omega)$ соболевскому пространству $H_{\mathrm{per}}^{1}(\square, d \mu)$, т.е. является непрерывной функцией на сетке $F$. Поскольку $\chi \in \mathscr{R}_{1}$, то условие закрепления должно выполняться.

3.5. ДоказАТЕЛЬСТво теоремы 3.2. По неравенству Юнга

$$
\int_{\Omega} A e\left(u^{\varepsilon, h}\right) \cdot e\left(u^{\varepsilon, h}\right) d \mu_{\varepsilon}^{h} \geqslant 2 \int_{\Omega} e\left(u^{\varepsilon, h}(x)\right) \cdot z(x) d \mu_{\varepsilon}^{h}-\int_{\Omega} A^{-1} z(x) \cdot z(x) d \mu_{\varepsilon}^{h}
$$

для любой симметрической матрицы $z \in L^{2}\left(\Omega, d \mu_{\varepsilon}^{h}\right)^{3}$. Возьмем

$$
z(x)=\Phi_{0}(x, y)+\frac{h}{\varepsilon} \sigma(y) \Phi_{1}(x, y), \quad y=\frac{x}{\varepsilon},
$$

где $\sigma(y)$ - матрица, определенная в $(3.12), \Phi_{0}, \Phi_{1}$ - пробные функции (матричная и скалярная соответственно) вида $\sum \varphi_{i}(x) b_{i}(y), \varphi_{i} \in C_{0}^{\infty}(\Omega), b_{i} \in C_{\mathrm{per}}^{\infty}(\square)$.

Предел первого слагаемого справа в (3.24) находим с использованием слабой двухмасштабной сходимости (3.2) и (3.13). В результате

$$
\lim _{\varepsilon \rightarrow 0} \int_{\Omega} e\left(u^{\varepsilon, h}\right) \cdot z d \mu_{\varepsilon}^{h}=\int_{\Omega} \int_{\square}\left(p \cdot \Phi_{0}+\frac{\theta^{2}}{3}(\chi \cdot \nu)^{\prime \prime} \Phi_{1}\right) d x d \mu .
$$

Учитывая конкретный вид матрицы $z$, имеем

$$
\begin{aligned}
\lim _{\varepsilon \rightarrow 0} \int_{\Omega} A^{-1} z \cdot z d \mu_{\varepsilon}^{h}= & \lim _{\varepsilon \rightarrow 0} \int_{\Omega} A^{-1} \Phi_{0} \cdot \Phi_{0} d \mu_{\varepsilon}^{h}+2 \lim _{\varepsilon \rightarrow 0} \frac{h}{\varepsilon} \int_{\Omega} A^{-1} \Phi_{0} \cdot \sigma \Phi_{1} d \mu_{\varepsilon}^{h} \\
& +\lim _{\varepsilon \rightarrow 0}\left(\frac{h}{\varepsilon}\right)^{2} \int_{\Omega} A^{-1} \sigma \cdot \sigma \Phi_{1}^{2} d \mu_{\varepsilon}^{h}=T_{1}+T_{2}+T_{3} .
\end{aligned}
$$

Величину $T_{1}$ находим по свойству среднего значения:

$$
T_{1}=\int_{\Omega} \int_{\square} A^{-1} \Phi_{0} \cdot \Phi_{0} d x d \mu
$$

Для выгисления величин $T_{2}$ и $T_{3}$ воспользуемся следующим утверждением. 
ПРЕДЛОЖЕНИЕ 3.8. Пусть $\beta=\beta^{h}(y)-$ функция, определенная в (3.12). Тогда при $\varepsilon \rightarrow 0$

$$
\beta\left(\frac{x}{\varepsilon}\right) \stackrel{2}{\rightarrow} 0, \quad\left(\beta\left(\frac{x}{\varepsilon}\right)\right)^{2} \stackrel{2}{\rightarrow} \frac{1}{3}
$$

Для доказательства нужно убедиться в том, что $\beta^{h} \rightarrow 0,\left(\beta^{h}\right)^{2} \rightarrow \frac{1}{3}$ в $L^{2}\left(\square, d \mu^{h}\right)$ и воспользоваться предложением 2.2 .

Из $(3.25)_{1}$ следует, что $T_{2}=0$. Вычислим теперь $T_{3}$. Заметим, что

$$
A^{-1} \sigma \cdot \sigma=\beta^{2} A^{-1} \eta \cdot \eta, \quad \eta=\tau \times \tau \text { на } h \text {-стержне с направлением } \tau \text {. }
$$

Поэтому из $(3.25)_{2}$ получаем, что

$$
T_{3}=\frac{\theta^{2}}{3} \int_{\Omega} \int_{\square} A^{-1} \eta \cdot \eta \Phi_{1}^{2} d x d \mu=\frac{\theta^{2}}{3} \int_{\Omega} \int_{\square} \rho^{-1} \Phi_{1}^{2} d x d \mu
$$

(см. (1.20)). В результате всех вычислений получаем неравенство

$$
\begin{aligned}
\liminf _{\varepsilon \rightarrow 0} \int_{\Omega} A e\left(u^{\varepsilon, h}\right) \cdot e\left(u^{\varepsilon, h}\right) d \mu_{\varepsilon}^{h} \geqslant & \int_{\Omega} \int_{\square}\left(2 p \cdot \Phi_{0}-A^{-1} \Phi_{0} \cdot \Phi_{0}\right) d x d \mu \\
& +\frac{\theta^{2}}{3} \int_{\Omega} \int_{\square}\left(2(\chi \cdot \nu)^{\prime \prime} \Phi_{1}-\rho^{-1} \Phi_{1}^{2}\right) d x d \mu .
\end{aligned}
$$

Берем здесь супремум по $\Phi_{0}$ и $\Phi_{1}$ и получаем искомое неравенство (3.3). Теорема 3.2 доказана.

\section{§4. Вывод усредненного уравнения}

4.1. Нам понадобятся некоторые сведения о пространстве $\mathscr{R}_{1}^{0}$ (см. определение 1.1).

Скажем, что вектор $g \in \mathscr{R}_{1}^{0}$ принадлежит множеству $D$, если

i) $g$ бесконечно дифференцируем вне некоторой окрестности узлов;

ii) в окрестности каждого узла он имеет структуру

$$
g(y)=C\left(\omega(y)-\omega\left(y^{0}\right)\right)
$$

где $C$ - константа, $y^{0}$ - узел, $\omega(y)=\left(-y_{2}, y_{1}\right)$.

Отметим, что $\omega$ - это жесткое перемещение на плоскости, т.е. $e(\omega)=0$. Подробнее условие (4.1) означает равенство $g(y) \cdot \nu_{i}=C\left(\omega(y)-\omega\left(y^{0}\right)\right) \cdot \nu_{i}$, где $\nu_{i}-$ нормали к выходящим из узла $y^{0}$ звеньям, а константа $C$ - одна и та же для всех $i$.

ПРЕДЛОЖЕНИЕ 4.1. Множество D плотно в пространстве $\mathscr{R}_{1}^{0}$. 
ДокАЗАТЕЛьСтво. Используя разложение единицы, легко заключить, что достаточно рассмотреть окрестность узла, поскольку гладкие функции плотны в пространстве $H^{2}(I)$, где $I$ - отрезок.

Пусть из узла $y^{0}=0$ выходит $m$ отрезков длины $\frac{1}{4}$. Имеем

$$
g(y) \cdot \nu_{i}=g_{i}(t), \quad t=\tau_{j} \cdot y, \quad 0 \leqslant t \leqslant \frac{1}{4} .
$$

По условию $g_{i} \in H^{2}\left(0, \frac{1}{4}\right)$ и

$$
g_{1}(0)=g_{2}(0)=\cdots=g_{m}(0)=0, \quad g_{1}^{\prime}(0)=g_{2}^{\prime}(0)=\cdots=g_{m}^{\prime}(0)=C .
$$

Очевидно, что функции $g_{i}(t)-C t$ можно аппроксимировать в пространстве $H^{2}\left(0, \frac{1}{4}\right)$ функциями, финитными около точки $t=0$. В результате сами функции $g_{i}(t)$ будут аппроксимированы функциями $\bar{g}_{i}(t)$, линейными около точки $t=0$.

Остается задать вектор $\bar{g}(y)$ равенством $\bar{g}(y) \cdot \nu_{i}=\bar{g}_{i}(t)$. В силу $(4.2)$ в некоторой окрестности узла $y^{0}=0$ имеем

$$
\bar{g}(y) \cdot \nu_{i}=C t=C \tau_{i} \cdot y=C \omega(y) \cdot \nu_{i}
$$

т.е. вьполнено равенство (4.1). Предложение доказано.

ЛЕмма 4.2. Для любого вектора $g \in D$ найдется его продолжение $g^{h}=$ $g^{h}(y)$ на сетку $F^{h}$ такое, что

i) $e_{y}\left(g^{h}\right)=0$ в окрестности каждого узла;

ii)

$$
A e_{y}\left(g^{h}\right)=h\left[(g \cdot \nu)^{\prime \prime} \rho\right]_{h} \sigma+O\left(h^{2}\right),
$$

əде $\sigma=\sigma(y)$ - матрица, определенная равенством $(3.12), \rho=\rho(y)$ скалярная функиия из $(1.20),\left[(g \cdot \nu)^{\prime \prime} \rho\right]_{h}$ - естественное продолэсение функиии $(g \cdot \nu)^{\prime \prime} \rho$ на $F^{h}$

iii) имеет место сильная сходимость

$$
g^{h} \rightarrow g \quad \text { в } \quad L^{2}\left(\square, d \mu^{h}\right)
$$

ДокАЗАТЕЛЬство. Рассмотрим узел $y_{0}=0$. В некоторой окрестности этого узла вектор $g$ совпадает с жестким перемешением (см. (4.1)). Определим $g^{h}$ этой же формулой и в $\delta$-окрестности точки $y_{0}=0$ на сетке $F^{h}$, т.е. положим

$$
g^{h}(y)=C \omega(y), \quad|y| \leqslant \delta, \quad y \in F^{h} .
$$

Теперь вектор $g$ надо продолжить на каждый $h$-стержень вне окрестности узла, причем это продолжение должно быть согласованным с (4.5).

Изучим сначала случай, когда тензор $A$ изотропен.

Рассмотрим горизонтальный стержень $I=[0, l]$. На нем вектор $g$ имеет вид

$$
g(y)=\left(0, a\left(y_{1}\right)\right), \quad a\left(y_{1}\right)=C y_{1}, \quad 0 \leqslant y_{1} \leqslant \delta
$$

где $C$ - та же константа, что и в (4.5). Продолжим этот вектор на стержень $I \times$ $[-h, h]$ равенством

$$
g^{h}(y)=\left(-y_{2} a^{\prime}\left(y_{1}\right), a\left(y_{1}\right)+k_{2} a^{\prime \prime}\left(y_{1}\right) \frac{y_{2}^{2}}{2}\right), \quad k_{2}=\frac{k_{1}}{k+k_{1}} .
$$


Данное продолжение согласовано с ранее построенным продолжением (4.5), поскольку на участке $[0, \delta] \times[-h, h]$ в силу (4.6) имеем

$$
g^{h}(y)=\left(-y_{2} C, C y_{1}\right)=C \omega(y) \text {. }
$$

Прямое вычисление показывает, что

$$
\begin{gathered}
e_{y}\left(g^{h}\right)=\left(\begin{array}{cc}
-y_{2} a^{\prime \prime}\left(y_{1}\right) & O\left(h^{2}\right) \\
O\left(h^{2}\right) & k_{2} y_{2} a^{\prime \prime}\left(y_{1}\right)
\end{array}\right) \\
A e_{y}\left(g^{h}\right)=\left(\begin{array}{cc}
-\widehat{k} y_{2} a^{\prime \prime}\left(y_{1}\right) & O\left(h^{2}\right) \\
O\left(h^{2}\right) & 0
\end{array}\right)=-\widehat{k} y_{2} a^{\prime \prime}\left(y_{1}\right)\left(\begin{array}{ll}
1 & 0 \\
0 & 0
\end{array}\right)+O\left(h^{2}\right) .
\end{gathered}
$$

Поскольку для горизонтального стержня

$$
\sigma(y)=-\frac{1}{h} y_{2}\left(\begin{array}{ll}
1 & 0 \\
0 & 0
\end{array}\right)
$$

то соотношение (4.3) выполнено (напомним, что для изотропного тензора $\rho=\widehat{k}$ ).

Аналогичное построение нетрудно провести и для наклонного стержня. В этом случае

$$
g(y)=\nu a(t), \quad t=\tau \cdot y, \quad a(t)=C t, \quad 0 \leqslant t \leqslant \delta,
$$

а продолжение на $h$-стержень определяем равенством

$$
g^{h}(y)=\nu\left(a(\tau \cdot y)+a^{\prime \prime}(\tau \cdot y) \frac{(\nu \cdot y)^{2}}{2} k_{2}\right)-\tau(\nu \cdot y) a^{\prime}(\tau \cdot y) .
$$

Это продолжение согласовано с продолжением (4.5), так как вблизи узла

$$
g^{h}(y)=C \nu(\tau \cdot y)-C \tau \nu \cdot y=C(\nu(\tau \cdot y)-\tau(\nu \cdot y))=C\left(-y_{2}, y_{1}\right)=C \omega(y) .
$$

Теперь построим продолжение в случае тензора $A$ общего вида.

Начнем с горизонтального отрезка $I=[0, l]$, где вектор $g$ имеет вид (4.6), и положим

$$
g^{h}(y)=\left(-y_{2} a^{\prime}\left(y_{1}\right)-\alpha a^{\prime \prime}\left(y_{1}\right) \frac{y_{2}^{2}}{2}, a\left(y_{1}\right)-\beta a^{\prime \prime}\left(y_{1}\right) \frac{y_{2}^{2}}{2}\right),
$$

где $\alpha, \beta$ - подлежащие определению константы. Это продолжение, действующее вне окрестности узлов, очевидно согласовано с продолжением (4.5). Так как

$$
-e_{y}\left(g^{h}\right)=\left(\begin{array}{cc}
y_{2} a^{\prime \prime}\left(y_{1}\right) & \alpha y_{2} a^{\prime \prime}\left(y_{1}\right) \\
\alpha y_{2} a^{\prime \prime}\left(y_{1}\right) & \beta y_{2} a^{\prime \prime}\left(y_{1}\right)
\end{array}\right)+O\left(h^{2}\right)=y_{2} a^{\prime \prime}\left(y_{1}\right)\left(\begin{array}{cc}
1 & \alpha \\
\alpha & \beta
\end{array}\right)+O\left(h^{2}\right),
$$

то для выполнения соотношения (4.3) требуется равенство

в котором

$$
A\left(\begin{array}{cc}
1 & \alpha \\
\alpha & \beta
\end{array}\right)=c\left(\begin{array}{ll}
1 & 0 \\
0 & 0
\end{array}\right)
$$

$$
c=\rho=\left(A^{-1} \eta \cdot \eta\right)^{-1}, \quad \eta=\left(\begin{array}{ll}
1 & 0 \\
0 & 0
\end{array}\right)
$$

(см. (1.20)). Определим матрицу $z=\left(\begin{array}{ll}\gamma & \alpha \\ \alpha & \beta\end{array}\right)$ равенством

$$
z=c A^{-1} \eta
$$

и убедимся, что $\gamma=1$. Действительно,

$$
\gamma=z \cdot \eta=c A^{-1} \eta \cdot \eta=1
$$

Тем самьм константы $\alpha, \beta$ однозначно определены и соотношение (4.3) обеспечено.

В случае наклонного стержня построение аналогично. Лемма полностью доказана. 
4.2. Установим следующую теорему усреднения.

ТЕОРема 4.3. Пусть $u^{\varepsilon, h}$ - решение задачи (2.1), причем последовательность правых частей $f^{\varepsilon, h}$ слабо двухмаситабно сходится

$$
f^{\varepsilon, h}(x) \stackrel{2}{\rightarrow} f(x, y)
$$

Тогда имеет место слабая двухмасштабная сходимость (3.1) и предельная функиия и есть решение усредненной задачи (1.17).

Если сходимость правых частей является сильной двухмасштабной

$$
f^{\varepsilon, h}(x) \stackrel{2}{\rightarrow} f(x, y)
$$

то сходимость решений становится такжсе сильной двухмасштабной. При этом имеет место сходимость упругих әнергий.

ДокАЗАТЕЛЬСтво. В работе [5] доказано, что (с точностью до выделения подпоследовательности)

$$
\begin{gathered}
e\left(u^{\varepsilon, h}\right) \stackrel{2}{\rightarrow} e\left(u^{0}\right)+v \\
v \in L^{2}\left(\Omega, \mathscr{V}_{\text {pot }}\right), \quad A\left(e\left(u^{0}\right)+v\right) \in L^{2}\left(\Omega, \mathscr{V}_{\text {sol }}\right)
\end{gathered}
$$

Здесь $\mathscr{V}_{\text {pot }}=\mathscr{V}_{\text {pot }}(\square, d \mu)-$ пространство периодических потенциальных матриц, определяемое как замыкание множества $\left\{e(\varphi), \varphi \in C_{\mathrm{per}}^{\infty}(\square)^{2}\right\}$ в $L^{2}(\square, d \mu)^{3}$, а $\mathscr{V}_{\text {sol }}=\mathscr{V}_{\text {sol }}(\square, d \mu)-$ пространство соленоидальных матрищ, $\mathscr{V}_{\text {sol }}=\left(\mathscr{V}_{\text {pot }}\right)^{\perp}$ в $L^{2}(\square, d \mu)^{3}$. Кроме того, доказано соотношение

$$
u^{0} \in H_{0}^{1}(\Omega)^{2}, \quad-\operatorname{div} A^{\mathrm{hom}} e\left(u^{0}\right)+\langle u\rangle=\langle f\rangle,
$$

совпадающее с (1.8) в том случае, когда $f$ не зависит от $y$. Оно получено некоторым предельным переходом в интегральном тождестве (2.1). Теперь необходимо сделать специальный предельный переход в этом тождестве с учетом особенностей критического случая.

В интегральном тождестве (2.1) возьмем пробную функцию

$$
\varphi(x)=w(x) g^{h}\left(\frac{x}{\varepsilon}\right),
$$

где $w \in C_{0}^{\infty}(\Omega), g^{h}(y)$ - продолжение на $F^{h}$ вектора $g(y) \in D$, рассмотренное в лемме 4.2. Тогда получим

$$
\begin{aligned}
\varepsilon^{-1} \int_{\Omega} A e\left(u^{\varepsilon, h}\right) & \cdot e_{y}\left(g^{h}\right) w d \mu_{\varepsilon}^{h}+\int_{\Omega} A e\left(u^{\varepsilon, h}\right) \cdot\left(g^{h} \times \nabla w\right) d \mu_{\varepsilon}^{h} \\
& =\int_{\Omega}\left(f^{\varepsilon, h}-u^{\varepsilon, h}\right) \cdot g^{h} w d \mu_{\varepsilon}^{h}
\end{aligned}
$$

Здесь при вычислении $e(\varphi)$ воспользовались равенством

$$
e\left(w g^{h}\right)=\varepsilon^{-1} w e_{y}\left(g^{h}\right)+\frac{1}{2}\left(g^{h} \times \nabla w+\nabla w \times g^{h}\right) .
$$


Предел правой части в (4.11) вычисляется просто. Действительно, из (4.4) и предложения 2.5 следует сильная сходимость $g^{h}(x / \varepsilon) \stackrel{2}{\rightarrow} g(y)$, которая вместе со слабой сходимостью $(3.1),(4.7)$ приводит к равенству

$$
\lim _{\varepsilon \rightarrow 0} \int_{\Omega}\left(f^{\varepsilon, h}-u^{\varepsilon, h}\right) \cdot g^{h} w d \mu_{\varepsilon}^{h}=\int_{\Omega} \int_{\square}(f-u) \cdot g w d x d \mu .
$$

Найдем предел первого слагаемого в левой части (4.11). Из (4.3) имеем

$$
\begin{aligned}
T_{1}(\varepsilon) & =\frac{1}{\varepsilon} \int_{\Omega} A e\left(u^{\varepsilon, h}\right) \cdot e_{y}\left(g^{h}\right) w d \mu_{\varepsilon}^{h}=\frac{1}{\varepsilon} \int_{\Omega} e\left(u^{\varepsilon, h}\right) \cdot A e_{y}\left(g^{h}\right) w d \mu_{\varepsilon}^{h} \\
& =\frac{h}{\varepsilon} \int_{\Omega} e\left(u^{\varepsilon, h}\right) \cdot \sigma\left(\frac{x}{\varepsilon}\right)\left[(g \cdot \nu)^{\prime \prime} \rho\right]_{h} w d \mu_{\varepsilon}^{h}+O(\varepsilon) .
\end{aligned}
$$

Слабая сходимость (3.13) и сильная сходимость $\left[(g \cdot \nu)^{\prime \prime} \rho\right]_{h} \stackrel{2}{\rightarrow}(g \cdot \nu)^{\prime \prime} \rho$ позволяют заключить, что

$$
\lim _{\varepsilon \rightarrow 0} T_{1}(\varepsilon)=\frac{\theta^{2}}{3} \int_{\Omega} \int_{\square} \rho(\chi \cdot \nu)^{\prime \prime}(g \cdot \nu)^{\prime \prime} w d x d \mu=\frac{\theta^{2}}{3} \int_{\Omega} \int_{\square} \rho \chi^{\prime \prime} \cdot g^{\prime \prime} w d x d \mu .
$$

Рассмотрим второе слагаемое в левой части (4.11). Из сильной двухмасштабной сходимости $g^{h}(x / \varepsilon) \stackrel{2}{\rightarrow} g(y)$ и слабой двухмасштабной сходимости (4.8) следует, что

$$
\lim _{\varepsilon \rightarrow 0} T_{2}(\varepsilon)=\int_{\Omega} \int_{\square} A\left(e\left(u^{0}(x)\right)+v(x, y)\right) \cdot(g(y) \times \nabla w) d x d \mu .
$$

Поскольку периодическая матрица $A\left(e\left(u^{0}(x)\right)+v(x, \cdot)\right)$ соленоидальна (см. $(4.9))$, то она поточечно ортогональна матрище $g(\cdot) \times \nabla w(x)$ (см. [5; лемма 5.3]). Это означает, что $\lim _{\varepsilon \rightarrow 0} T_{2}(\varepsilon)=0$. Итак, установлено тождество

$$
\frac{\theta^{2}}{3} \int_{\Omega} \int_{\square} \rho \chi^{\prime \prime} \cdot \psi^{\prime \prime} d x d \mu=\int_{\Omega} \int_{\square}(f-u) \cdot \psi d x d \mu
$$

в котором $\psi(x, y)=w(x) g(y), g \in D$. Отсюда заключаем (в силу предложения 4.1), что это тождество вьполнено для любого $\psi \in L^{2}\left(\Omega, \mathscr{R}_{1}^{0}\right)$.

Рассмотрим произвольный элемент энергетического пространства $V$,

$$
\varphi(x, y)=\varphi^{0}(x)+\psi(x, y), \quad \varphi^{0} \in H_{0}^{1}(\Omega)^{2}, \quad \psi \in L^{2}\left(\Omega, \mathscr{R}_{1}^{0}\right) .
$$

Из (4.10) следует, что

$$
\int_{\Omega} A^{\mathrm{hom}} e\left(u^{0}\right) \cdot e\left(\varphi^{0}\right) d x+\int_{\Omega} \int_{\square} u \cdot \varphi^{0} d x d \mu=\int_{\Omega} \int_{\square} f \cdot \varphi^{0} d x d \mu .
$$

Если теперь сложить это тождество с тождеством (4.12), то получим, что $u$ есть решение усредненного уравнения (1.17). Этим исчерпан случай слабой сходимости правых частей. 
Изучим случай сильной сходимости $f^{\varepsilon, h}(x) \stackrel{2}{\rightarrow} f(x, y)$. Для этого рассмотрим второй вариант задачи (2.1), в котором решение обозначено через $v^{\varepsilon, h}$, а правая часть - через $z^{\varepsilon, h}$, т.е.

$v^{\varepsilon, h} \in W_{\varepsilon, h}, \quad \int_{\Omega}\left(A e\left(v^{\varepsilon, h}\right) \cdot e(\varphi)+v^{\varepsilon, h} \cdot \varphi\right) d \mu_{\varepsilon}^{h}=\int_{\Omega} z^{\varepsilon, h} \cdot \varphi d \mu_{\varepsilon}^{h} \forall \varphi \in C_{0}^{\infty}(\Omega)^{2}$.

Полагая здесь $\varphi=u^{\varepsilon, h}$, а в $(2.1) \varphi=v^{\varepsilon, h}$, получаем формулу Грина-Бетти

$$
\int_{\Omega} u^{\varepsilon, h} \cdot z^{\varepsilon, h} d \mu_{\varepsilon}^{h}=\int_{\Omega} v^{\varepsilon, h} \cdot f^{\varepsilon, h} d \mu_{\varepsilon}^{h}
$$

Считаем, что $z^{\varepsilon, h} \stackrel{2}{\rightarrow} z$. Тогда

$$
\lim _{\varepsilon \rightarrow 0} \int_{\Omega} u^{\varepsilon, h} \cdot z^{\varepsilon, h} d \mu_{\varepsilon}^{h}=\int_{\Omega} \int_{\square} v \cdot f d x d \mu=\int_{\Omega} \int_{\square} u \cdot z d x d \mu .
$$

Здесь мы воспользовались сильной сходимостью $f^{\varepsilon, h} \stackrel{2}{\rightarrow} f($ см. $(2.7))$, слабой сходимостью $v^{\varepsilon, h} \stackrel{2}{\rightarrow} v$, где $v$ - решение усредненного уравнения, а также формулой Грина-Бетти, связываюшей решения $u, v$ усредненного уравнения. Поскольку $z^{\varepsilon, h}$ - произвольная слабо двухмасштабно сходящаяся последовательность, то равенство (4.13) дает искомую сильную двухмасштабную сходимость $u^{\varepsilon} \stackrel{2}{\rightarrow} u$ (см. (2.7)).

Сходимость энергий устанавливается теперь совсем просто. Из (2.2) и (2.8) имеем

$$
\lim _{\varepsilon \rightarrow 0} \int_{\Omega} A e\left(u^{\varepsilon, h}\right) \cdot e\left(u^{\varepsilon, h}\right) d \mu_{\varepsilon}^{h}+\int_{\Omega} \int_{\square} u \cdot u d x d \mu=\int_{\Omega} \int_{\square} f \cdot u d x d \mu .
$$

Сравнивая это равенство с энергетическим равенством (1.18), получаем (1.19). Теорема доказана.

Теперь обратимся к исходной задаче (1.2) с гладкой правой частью $f$. Компонента $u^{0}$ является решением эллиптической задачи $(1.15)_{1}$ и поэтому $u^{0} \in C(\bar{\Omega})^{2}$. Тог да из представления $(1.15)_{2}$ следует, что выполнены условия леммы 2.6 и сильная сходимость $u^{\varepsilon, h} \stackrel{2}{\rightarrow} u$ влечет соотношение (1.6). Тем самым и теорема 1.3 доказана.

Наконец рассмотрим задачу теории упругости в статической постановке. Запишем уравнение (1.21) в виде

$$
u^{\varepsilon, h} \in W_{\varepsilon, h}, \quad \int_{\Omega} A e\left(u^{\varepsilon, h}\right) \cdot e(\varphi) d \mu_{\varepsilon}^{h}=\int_{\Omega} f^{\varepsilon, h} \cdot \varphi d \mu_{\varepsilon}^{h}, \quad \varphi \in C_{0}^{\infty}(\Omega)^{2},
$$

где правая часть $f^{\varepsilon, h}$ зависит от $\varepsilon, h$.

Теорема 4.4. Пусть $f^{\varepsilon, h}(x) \stackrel{2}{\rightarrow} f(x, y)$. Тогда имеет место слабая двухмаситабная сходимость (3.1) и предельная функиия $u(x, y)=u^{0}(x)+\chi(x, y)$ есть решение усредненной задачи

$$
\begin{gathered}
u \in V, \quad \int_{\Omega} A^{\mathrm{hom}} e\left(u^{0}\right) \cdot e\left(\varphi^{0}\right) d x+\frac{\theta^{2}}{3} \int_{\Omega} \int_{\square} \rho \chi^{\prime \prime} \cdot \psi d x d \mu=\int_{\Omega} \int_{\square} f \cdot \varphi d x d \mu \\
\forall \varphi=\varphi^{0}+\psi \in V .
\end{gathered}
$$

Если сходимость правых частей является сильной двухмасштабной, т.е. $f^{\varepsilon, h}(x) \stackrel{2}{\rightarrow} f(x, y)$, то сходимость решений становится такжсе сильной двухмаситабной. При этом имеет место сходимость упругих энергий. 
ДоКАЗАТЕЛЬСТво. В силу неравенства Корна (1.22) для решений $u^{\varepsilon, h}$ выполнено условие ограниченности (2.3). Без потери общности считаем, что имеет место сходимость (3.1), причем $u \in V$ по теореме 3.1.

Формально задачу (4.15) можно записать в виде (2.1), а именно,

$$
u^{\varepsilon, h} \in W_{\varepsilon, h}, \quad \int_{\Omega}\left(A e\left(u^{\varepsilon, h}\right) \cdot e(\varphi)+u^{\varepsilon, h} \cdot \varphi\right) d \mu_{\varepsilon}^{h}=\int_{\Omega} g^{\varepsilon, h} \cdot \varphi d \mu_{h}^{\varepsilon},
$$

где $g^{\varepsilon, h}=u^{\varepsilon, h}+f^{\varepsilon, h}$. Поскольку $g^{\varepsilon, h}(x) \stackrel{2}{\rightarrow} g(x, y)=u(x, y)+f(x, y)$, то по теореме 4.3 предельная функция $u$ есть решение усредненной задачи (4.15). Этим исчерпан случай слабой сходимости правых частей.

Сильная сходимость $u^{\varepsilon, h} \stackrel{2}{\rightarrow} u$, а также сходимость энергий устанавливаются в точности так же, как и в теореме 4.3. Теорема доказана.

\section{§5. Приложение: некоторые вспомогательные вопросы}

5.1. Начнем с некоторых неравенств в единичном квадрате $\square=(0,1)^{2}$.

Лемма 5.1. Справедливо неравенство

$$
\int_{\square}|\nabla v|^{2} d y \leqslant c\left(\int_{\square} e(v) \cdot e(v) d y+\int_{\square}\left|v_{2}\right|^{2} d y\right), \quad v \in C^{\infty}(\square)^{2} .
$$

ДокАЗАТЕльство. Допуская, что эта оценка неверна, найдем гладкие векторы $v^{k}$ такие, что

$$
\int_{\square}\left|\nabla v^{k}\right|^{2} d y=1, \quad \int_{\square}\left(e\left(v^{k}\right) \cdot e\left(v^{k}\right)+\left|v_{2}^{k}\right|^{2}\right) d y \rightarrow 0 .
$$

Без потери общности предполагаем, что $\int_{\square} v_{1}^{k} d y=0$. Последовательность $v^{k}$ ограничена в $H^{1}(\square)^{2}$ и можно считать ее слабо сходящейся, т.е. $v^{k} \rightarrow v, \nabla v^{k} \rightarrow \nabla v$ в $L^{2}(\square)$. Тогда из (5.2) имеем

$$
e(v)=0, \quad v_{2}=0, \quad \int_{\square} v_{1} d y=0 .
$$

Отсюда получаем, что $v=0$. С другой стороны, из классического неравенства Корна

$$
\int_{\square}|\nabla v|^{2} d y \leqslant c_{1} \int_{\square}(e(v) \cdot e(v)+v \cdot v) d y
$$

следует, что $\int_{\square}\left|v^{k}\right|^{2} \geqslant c_{1} / 2>0$ при достаточно большом $k$, что противоречит сильной сходимости $v^{k} \rightarrow 0$ в $L^{2}(\square)$. Лемма доказана. 


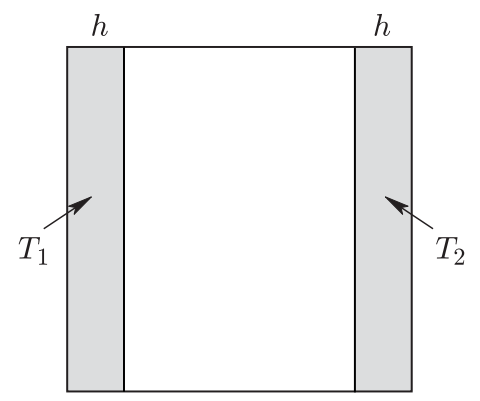

Рис. 4.

ЛЕМма 5.2. Пусть $T_{1}, T_{2}$ - две полосы ширины $h$, примыкающие $к$ левой и правой сторонам квадрата $\square$ (см. рис. 4). Тогда

$$
\int_{\square}|\nabla v|^{2} d y \leqslant c\left(\int_{\square} e(v) \cdot e(v) d y+\frac{1}{h} \sum_{i=1}^{2} \int_{T_{i}}\left|v_{2}\right|^{2} d y\right), \quad v \in C^{\infty}(\square)^{2},
$$

причем константа с не зависит от $h$.

Доказывается эта лемма тем же способом, что и лемма 5.1, с использованием стандартных свойств следа функций из $H^{1}(\square)$.

Ниже удобно считать, что стержни (полосы) имеют толщину $h$, а не $2 h$, как это было в основном тексте.

Рассмотрим полосу $I^{h}=[0,1] \times[0, h]$.

Делаем стандартную в теории пластин замену

$$
x_{1}=y_{1}, \quad x_{2}=h y_{2}, \quad u_{1}=v_{1}, \quad u_{2}=h^{-1} v_{2},
$$

в результате которой неравенство (5.1) примет вид

$$
\begin{aligned}
& \int_{I^{h}}\left(\left(\frac{\partial u_{1}}{\partial x_{1}}\right)^{2}+h^{2}\left(\frac{\partial u_{1}}{\partial x_{2}}\right)^{2}+h^{2}\left(\frac{\partial u_{2}}{\partial x_{1}}\right)^{2}+h^{4}\left(\frac{\partial u_{2}}{\partial x_{2}}\right)^{2}\right) d x \\
& \leqslant c \int_{I^{h}}\left(\left(\frac{\partial u_{1}}{\partial x_{1}}\right)^{2}+\frac{h^{2}}{2}\left(\frac{\partial u_{1}}{\partial x_{2}}+\frac{\partial u_{2}}{\partial x_{1}}\right)^{2}+h^{4}\left(\frac{\partial u_{2}}{\partial x_{2}}\right)^{2}+h^{2} u_{2}^{2}\right) d x
\end{aligned}
$$

Отсюда

$$
h^{2} \int_{I^{h}}\left(\left(\frac{\partial u_{1}}{\partial x_{2}}\right)^{2}+\left(\frac{\partial u_{2}}{\partial x_{1}}\right)^{2}\right) d x \leqslant c \int_{I^{h}}\left(e(u) \cdot e(u)+h^{2} u_{2}^{2}\right) d x
$$

а поскольку

$$
\int_{I^{h}}\left(\left(\frac{\partial u_{1}}{\partial x_{1}}\right)^{2}+\left(\frac{\partial u_{2}}{\partial x_{2}}\right)^{2}\right) d x \leqslant \int_{I^{h}} e(u) \cdot e(u) d x
$$

то получаем оценку

$$
h^{2} \int_{I^{h}}|\nabla u|^{2} d x \leqslant c \int_{I^{h}}\left(e(u) \cdot e(u)+h^{2} u_{2}^{2}\right) d x .
$$


Для полосы $\varepsilon I^{h}=[0, \varepsilon] \times[0, \varepsilon h]$ соответствующая оценка, получающаяся гомотетией, имеет вид

$$
h^{2} \int_{\varepsilon I^{h}}|\nabla u|^{2} d x \leqslant c \int_{\varepsilon I^{h}}\left(e(u) \cdot e(u)+\left(\frac{h}{\varepsilon}\right)^{2} u_{2}^{2}\right) d x .
$$

Из этой оценки следует оценка (3.23), использованная для вывода условий закрепления в узлах. Действительно, достаточно просуммировать оценки вида (5.5) по всем элементарным полоскам $\varepsilon I^{h}$, которые принадлежат $\Omega \cap F_{\varepsilon}^{h}$.

5.2. Из оценки (5.3), сделав согласованную замену (5.4) функции $v$ на функцию $u$ и переменной $y$ на переменную $x$, вьводим оценку на полосе $I^{h}$ :

$$
h^{2} \int_{I^{h}}|\nabla u|^{2} d x \leqslant c\left(\int_{I^{h}} e(u) \cdot e(u) d x+h \sum_{i=1}^{2} \int_{Q_{h}^{i}}\left|u_{2}\right|^{2} d x\right),
$$

где $Q_{n}^{1}, Q_{h}^{2}$ - квадраты со стороной $h$, примыкаюшие к торцам полосы $I^{h}$ (см. рис. 5).

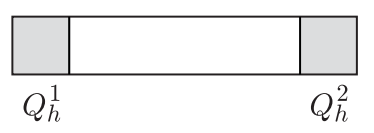

Рис. 5.

Отсюда гомотетией получаем оценку

$$
h^{2} \int_{\varepsilon I^{h}}|\nabla u|^{2} d x \leqslant c\left(\int_{\varepsilon I^{h}} e(u) \cdot e(u) d x+\frac{h}{\varepsilon^{2}} \sum_{i=1}^{2} \int_{Q_{\varepsilon h}^{i}}\left|u_{2}\right|^{2} d x\right) .
$$

Нам понадобятся также некоторые элементарные вариации неравенства Фридрихса для скалярных функций.

i) Для гладкой в полосе $I^{h}$ функции $w$ вьполнена оценка

$$
\int_{I^{h}}|w|^{2} d x \leqslant c\left(\int_{I^{h}}\left|\frac{\partial w}{\partial x_{1}}\right|^{2} d x+\frac{1}{h} \sum_{i=1}^{2} \int_{Q_{h}^{i}}|w|^{2} d x\right)
$$

из которой гомотетией получаем оценку

$$
\int_{\varepsilon I^{h}}|w|^{2} d x \leqslant c\left(\varepsilon^{2} \int_{\varepsilon I^{h}}\left|\frac{\partial w}{\partial x_{1}}\right|^{2} d x+\frac{1}{h} \sum_{i=1}^{2} \int_{Q_{\varepsilon h}^{i}}|w|^{2} d x\right) .
$$

(В этой оценке в отличие от (5.6) можно ограничиться одним из двух квадратов.)

ii) Если $P$ - горизонтальная полоса ширины $t$, пересекаюшая область $\Omega$, то для $w \in C_{0}^{\infty}(\Omega)$

$$
\int_{P}|w|^{2} d x \leqslant d \int_{P}\left|\frac{\partial w}{\partial x_{1}}\right|^{2} d x
$$

где $d$ - диаметр области $\Omega$. Кроме того, для любого квадрата $Q_{t}$ со стороной $t$, принадлежащего полосе $P$,

$$
\int_{Q_{t}}|w|^{2} d x \leqslant t d \int_{P}\left|\frac{\partial w}{\partial x_{1}}\right|^{2} d x
$$


5.3. Приступим непосредственно к доказательству неравенства Корна (1.22) для модельной сетки. Для ясности проведем все рассуждения для случая квадратной сетки.

Квадратная сетка составлена из горизонтальных полос $P=P_{j}$ и вертикальных полос $S=S_{j}$, имеющих толщину $t=\varepsilon h$. Эти полосы пересекаются по квадратам $Q_{t}$ со стороной $t=\varepsilon h$.

Рассмотрим отдельную горизонтальную полосу $P$. Для компоненты $u_{1}$ по неравенству Фридрихса имеем

$$
\int_{P}\left|u_{1}\right|^{2} d x \leqslant d \int_{P}\left|\frac{\partial u_{1}}{\partial x_{1}}\right|^{2} d x \leqslant d \int_{P} e(u) \cdot e(u) d x
$$

Чтобы оценить компоненту $u_{2}$, выделим на полосе фрагмент типа $\varepsilon I^{h}$ (см. рис. 6 ).

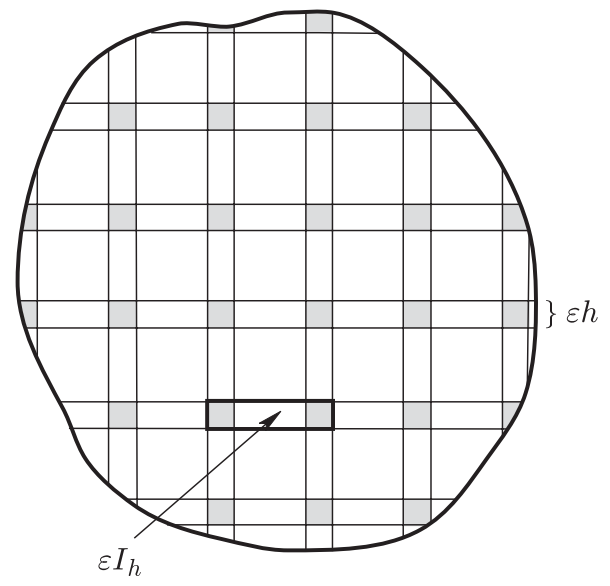

Рис. 6.

Из (5.7), (5.6) получим

$$
\begin{aligned}
\int_{\varepsilon I^{h}}\left|u_{2}\right|^{2} d x & \leqslant c\left(\varepsilon^{2} \int_{\varepsilon I^{h}}\left|\frac{\partial u_{2}}{\partial x_{1}}\right|^{2} d x+\frac{1}{h} \sum_{i=1}^{2} \int_{Q_{\varepsilon h}^{i}}\left|u_{2}\right|^{2} d x\right) \\
& \leqslant c_{1}\left(\left(\frac{\varepsilon}{h}\right)^{2} \int_{\varepsilon I^{h}} e(u) \cdot e(u) d x+\frac{1}{h} \sum_{i=1}^{2} \int_{Q_{\varepsilon h}^{i}}\left|u_{2}\right|^{2} d x\right) .
\end{aligned}
$$

Согласно оценке вида (5.9) для вертикальных полос, пересекающих $Q_{\varepsilon h}^{1}, Q_{\varepsilon h}^{2}$, и функции $w=u_{2}$ имеем

$$
\frac{1}{h} \int_{Q_{\varepsilon h}^{1}}\left|u_{2}\right|^{2} d x+\frac{1}{h} \int_{Q_{\varepsilon h}^{2}}\left|u_{2}\right|^{2} d x \leqslant 2 d \varepsilon\left(\int_{S_{1}} e(u) \cdot e(u) d x+\int_{S_{2}} e(u) \cdot e(u) d x\right),
$$

где $S_{1}, S_{2}$ - соответствуюшие вертикальные полосы. Отсюда суммированием по всем фрагментам типа $\varepsilon I^{h}$, на которые можно разбить полосу $P$, получаем

$$
\int_{P}\left|u_{2}\right|^{2} d x \leqslant c_{2}\left(\int_{P} e(u) \cdot e(u) d x+\varepsilon \int_{\Omega} e(u) \cdot e(u) d x\right) .
$$


Теперь просуммируем полученные неравенства по всем горизонтальным полоскам и учтем, что число этих полосок имеет порядок $\varepsilon^{-1}$. В результате получим оценку

$$
\sum \int_{P_{j}}\left|u_{2}\right|^{2} d x \leqslant c_{2} \int_{\Omega} e(u) \cdot e(u) d x .
$$

Отсюда и из (5.10), а также аналогичных оценок для вертикальных полос $S_{j}$ следует неравенство (1.22).

Отметим, что много ценного материала по неравенствам Корна и вообще задачам теории упругости на стержневых структурах имеется в работах С. А. Назарова (см. [6]-[8]). Разнообразные неравенства Корна на тонких структурах, в том числе те, что использованы при изучении задач $(1.21),(2.1)$, можно найти в работе авторов [9].

5.4. Перейдем к обсуждению теоремњ 3.7 из теории двухмасштабной сходимости.

Если $\mu$-периодическая мера в $\mathbb{R}^{N}$, то соотношение $\operatorname{div} b=a$ (в смысле меры $\mu$ ) означает, что $a \in L^{2}(\square, d \mu), b \in L^{2}(\square, d \mu)^{N}$ и

$$
\int_{\square} b \cdot \nabla \varphi d \mu=-\int_{\square} a \varphi d \mu, \quad \varphi \in C_{\mathrm{per}}^{\infty}(\square) .
$$

В частности, если $a=0$, то вектор $b$ соленоидален, $b \in \mathscr{V}_{\text {sol }}(\square, d \mu)$.

Пусть $\mu^{h}, \mu$-периодические меры, $\mu^{h} \rightarrow \mu$. Напомним (см. [5]) так назьваемые аппроксимативные условия.

i) Для любой функции $а$ из некоторого плотного в $L^{2}(\square, d \mu) / \mathbb{R}^{1}$ множества найдутся $a^{h} \in L^{2}\left(\square, d \mu^{h}\right), b^{h} \in L^{2}(\square, d \mu)^{N}$ такие, что $\operatorname{div} b^{h}=a^{h}$ (в смысле меры $\left.\mu^{h}\right), a^{h} \rightarrow a, b^{h} \rightarrow b$ в $L^{2}\left(\square, d \mu^{h}\right)$.

ii) Для любого вектора $b \in \mathscr{V}_{\text {sol }}(\square, d \mu)$ найдется вектор $b^{h} \in \mathscr{V}_{\text {sol }}\left(\square, d \mu^{h}\right)$ такой, что $b^{h} \rightarrow b$ в $L^{2}\left(\square, d \mu^{h}\right)$ (сильная аппроксимируемость соленоидальных векторов).

Имеет место следующая

Теорема 5.3. Пусть $\mu^{h}, \mu$ - периодические борелевы меры, $\mu^{h} \rightarrow \mu$, которые связаны аппроксимативными условиями і), iі). Пусть, кроме того, для предельной меръ $\mu$ справедливо неравенство Пуанкаре

$$
\int_{\square} \varphi^{2} d \mu \leqslant C \int_{\square}|\nabla \varphi|^{2} d \mu, \quad \varphi \in C_{\mathrm{per}}^{\infty}(\square), \quad \int_{\square} \varphi d \mu=0 .
$$

Тогда для последовательности скалярных функций $v^{\varepsilon, h}$, удовлетворяющей условию ограниченности (3.21), въполнено свойство (3.22).

В классическом случае, когда $d \mu^{h}=d y$ - мера Лебега, эта теорема доказана в [10], [11].

Случай постоянной борелевой меры $\left(\mu^{h}=\mu\right)$ рассмотрен в [12; теорема 4.5]. Доказательство обшей версии проводится аналогично с использованием аппроксимативных условий.

5.5. Обратимся непосредственно к теореме 3.7. Неравенство Пуанкаре (5.11) есть следствие связности сингулярной сетки. Поэтому остается проверить аппроксимативные условия i), ii). 
Начнем с проверки условия іi). Пусть $b$ - соленоидальный вектор на сетке $F$, $b \in \mathscr{V}_{\text {sol }}(\square, d \mu)$. Непосредственно из определения

$$
\int_{\square} b \cdot \nabla \varphi d \mu=0 \quad \forall \varphi \in C_{\mathrm{per}}^{\infty}(\square)
$$

легко получить (см. $[11 ; \S 9])$ условия, которым вектор $b$ удовлетворяет на каждом звене и в каждом узле сетки $F$.

Пусть $I_{1}, \ldots, I_{m}$ - все звенья, сходящиеся в узле $O, \tau_{1}, \ldots, \tau_{m}$ - единичные направляющие векторы этих звеньев, выбранные выходящими из узла. Тогда

$$
\begin{gathered}
\left.b\right|_{I_{i}}=b_{i} \tau_{i}, \quad b_{i}=\text { const } \\
\sum_{i=1}^{m} b_{i}=0
\end{gathered}
$$

Для построения вектора $b^{h} \in V_{\text {sol }}\left(\square, d \mu^{h}\right)$ решим вспомогательную задачу в некоторой окрестности $Q_{h}$ каждого узла $O$. Определим окрестность $Q_{h}$ как объединение круга радиуса $h$ с центром в узле $O$ и $m$ полос, у которых ширина $-2 h$, длина $-4 h$, а средняя линия лежит на $I_{i}$, и пусть $\Gamma_{1}, \ldots, \Gamma_{m}$ - внешние торцы этих полос (см. рис. 7 ). В области $Q_{h}$ рассмотрим следующую задачу Неймана

$$
\begin{gathered}
\operatorname{div}(\nabla u)=0 \quad \text { в } \quad Q_{h} ;\left.\quad \frac{\partial u}{\partial n}\right|_{\Gamma_{i}}=b_{i}, \quad i=1, \ldots, m ; \\
\frac{\partial u}{\partial n}=0 \quad \text { на остальной части } \partial Q_{h},
\end{gathered}
$$

где $n$ - единичная внешняя нормаль к $\partial Q_{h}$.

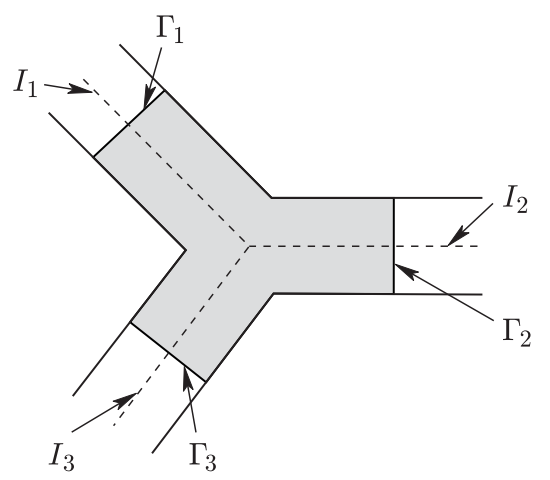

Рис. 7. Область $Q_{h}$

Разрешимость этой задачи следует из (5.12), и несложные вычисления приводят к оценке

$$
\int_{Q_{h}}|\nabla u|^{2} d x \leqslant c h^{2} .
$$

Положим

$$
b^{h}= \begin{cases}\nabla u & \text { в } Q_{h} \\ b_{h} & \text { вне } Q_{h}\end{cases}
$$


где $b_{h}$ - естественное продолжение вектора $b$. Тогда $b^{h} \in \mathscr{V}_{\text {sol }}\left(\square, d \mu^{h}\right)$ и $b^{h} \rightarrow b$ в $L^{2}\left(\square, d \mu^{h}\right)$.

Тем самьм условие іi) проверено.

Обратимся к проверке условия і).

Легко видеть, что в пространстве $L^{2}(\square, d \mu) / \mathbb{R}^{1}$ плотны гладкие функции, равные нулю в окрестности узлов. Пусть $a$ - такая периодическая функция. Из неравенства Пуанкаре (5.11) следует, что задача

$$
v \in H_{\mathrm{per}}^{1}(\square, d \mu), \quad \operatorname{div} \nabla v=a
$$

имеет решение. Положим $b=\nabla v$.

Вне области $Q_{h}$ функцию $a$ и вектор $b$ продолжим естественным образом. В окрестности узлов вектор $b$ соленоидален, и его можно продолжить в область $Q_{h}$ указанным выше способом.

Отметим, что вопросы, связанные с аппроксимативными свойствами, рассматривались в работе [13].

\section{Список литературы}

1. Бахвалов Н. С., Панасенко Г. П. Осреднение процессов в периодических средах. М.: Наука, 1984.

2. ЖКиков В. В., Козлов С. М., Олейник О.А. Усреднение дифференциальных операторов. М.: Наука, 1993.

3. Олейник O. А., Иосифьян Г. А., Шамаев А. С. Математические задачи теории сильно неоднородных упругих сред. М.: Изд-во МГУ, 1990.

4. Жиков В. В. Усреднение задач теории упругости на сингулярных структурах // Докл. AH. 2001. T. 380. №6. C. 741-745.

5. Жиков В. В. Усреднение задач теории упругости на сингулярных структурах // Изв. РАН. Сер. матем. 2002. Т. 66. № 2. С. 81-148.

6. Nazarov S. A. Korn's inequalities for functions of spatial bodies and thin rods // Math. Methods Appl. Sci. 1997. V. 20. №3. P. 219-243.

7. Назаров С. A., Слуцкий A. С. Произвольные плоские системы анизотропных балок // Труды МИРАН. 2002. Т. 236. С. 234-261.

8. Назаров С.А. Неравенства Корна, асимптотически точные для тонких областей // Вестн. СПбГУ. Сер. 1. 1992. Т. 2. № 8. С. 19-24.

9. Жиков В. В., Пастухова C. E. О неравенствах Корна на тонких периодических структурах // Докл. РАН. 2003. V. 388. № 5. Р. 588-592.

10. Nguetseng $G$. A general convergence result for a functional related to the theory of homogenization // SIAM J. Math. Anal. 1989. V. 20. P. 608-623.

11. Allaire G. Homogenization and two-scale convergence // SIAMJ. Math. Anal. 1992. V. 23. P. $1482-1518$.

12. Жиков В.В. Об одном расширении и применении метода двухмасштабной сходимости // Матем. сб. 2000. Т. 191. № 7. С. 31-72.

13. Chechkin G.A., Jikov V.V., Lukkassen D., Piatnitski A.L. On homogenization of networks and junctions // Asymptot. Anal. 2002. V. 30. № 1. P. 61-80.

Владимирский государственньй педагогический университет;

Московский институт радиотехники, электроники и автоматики

E-mail: zhikov@vgpu.vladimir.ru, leonowmw@cs.msu.su
Поступила в редакцию

03.06.2002 